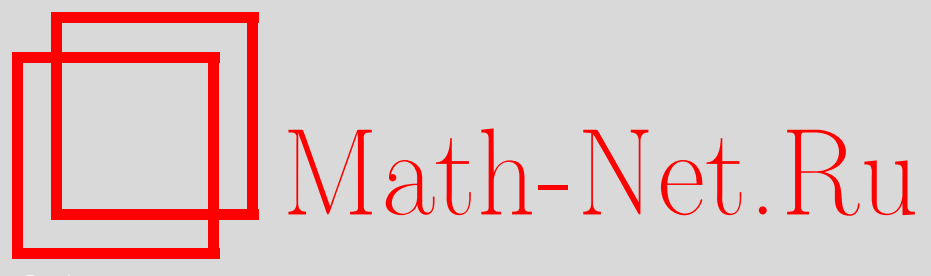

А. Ю. Колесов, Н. Х. Розов, В. А. Садовничий, О проблеме возникновения автоволн в параболических системах с малой диффузией, Матем. сб., 2007, том 198, номер 11, 67-106

DOI: https://doi.org/10.4213/sm3792

Использование Общероссийского математического портала Math-Net.Ru подразумевает, что вы прочитали и согласны с пользовательским соглашением http://www . mathnet.ru/rus/agreement

Параметры загрузки:

IP: 54.210 .77 .194

26 апреля 2023 г., 15:18:22

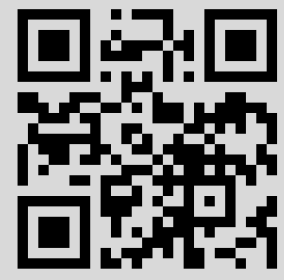




\author{
А. Ю. Колесов, Н. Х. Розов, В. А. Садовничий
}

\title{
О проблеме возникновения автоволн в параболических системах с малой диффузией
}

\begin{abstract}
Рассматривается параболическая система типа реакция-диффузия с нулевыми граничными условиями Неймана на концах конечного отрезка при следующих основных предположениях. Во-первых, считаем, что матричный коэффициент диффузии в ней пропорционален некоторому малому параметру $\varepsilon>0$, а сама она имеет пространственно однородный (не зависящий от пространственной переменной) цикл амплитуды порядка $\sqrt{\varepsilon}$, родившийся из нулевого состояния равновесия в результате бифуркации Андронова-Хопфа. Во-вторых, предполагаем, что матричная диффузия зависит от дополнительного малого параметра $\mu \geqslant 0$ и при $\mu=0$ в задаче об устойчивости однородного цикла реализуется критический случай двукратного единичного мультипликатора без жордановой клетки. При перечисленных ограничениях и при независимом изменении параметров $\varepsilon$ и $\mu$ исследуется вопрос о существовании и устойчивости пространственно неоднородных автоколебаний, ответвляющихся от однородного цикла.

Библиография: 16 названий.
\end{abstract}

\section{§ 1. Постановка задачи и предварительные результаты}

1.1. Описание объекта исследования. На отрезке $0 \leqslant x \leqslant \pi$ рассмотрим краевую задачу

$$
\frac{\partial u}{\partial t}=\varepsilon D \frac{\partial^{2} u}{\partial x^{2}}+A(\varepsilon) u+F(u, \varepsilon),\left.\quad \frac{\partial u}{\partial x}\right|_{x=0}=\left.\frac{\partial u}{\partial x}\right|_{x=\pi}=0
$$

с малым параметром $\varepsilon>0$. Здесь $u \in \mathbb{R}^{N}, N \geqslant 2, D$ - матрица диффузии (которая в дальнейшем будет зависеть еще от другого малого параметра $\mu$ ), а бесконечно дифференцируемые по $\varepsilon \in\left[0, \varepsilon_{0}\right], \varepsilon_{0}=$ const $>0$, и $(u, \varepsilon) \in \mathbb{R}^{N} \times\left[0, \varepsilon_{0}\right]$ соответственно квадратная матрица $A(\varepsilon)$ и вектор-функция $F(u, \varepsilon)$ удовлетворяют ряду дополнительных ограничений.

Сформулируем сначала группу условий, относящихся к матрице $A(\varepsilon)$. С этой целью введем в рассмотрение матрицу $A_{0}=A(0)$ и предположим, что она имеет простую пару чисто мнимых собственных значений $\lambda= \pm i \omega_{0}, \omega_{0}>0$, которым отвечают собственные векторы $a$ и $\bar{a}$, нормированные условиями $(a, b)=1$, $(\bar{a}, b)=0$, где $A_{0}^{*} b=-i \omega_{0} b$, а через $(*, *)$ здесь и ниже будем обозначать евклидово скалярное произведение. Что же касается остальных ее собственных значений, то их считаем лежащими в комплексной полуплоскости $\{\lambda: \operatorname{Re} \lambda<0\}$.

Работа выполнена при финансовой поддержке Российского фонда фундаментальных исследований (грант № 05-01-01004) и Целевой программы "Развитие научного потенциала высшей школы" (грант № РНП.2.1.1.630).

(С А. Ю. Колесов, Н. Х. Розов, В. А. САдовничий, 2007 
Кроме того, предполагаем выполненным неравенство

$$
\phi_{0}=\operatorname{Re}\left(A_{1} a, b\right)>0,
$$

где $A_{1}=\left.\frac{d}{d \varepsilon} A(\varepsilon)\right|_{\varepsilon=0}$, означающее, что при малых $\varepsilon>0$ упомянутые выше чисто мнимые собственные значения сдвигаются в правую комплексную полуплоскость.

Обратимся, далее, к фигурирующей в (1.1) нелинейности $F$. Будем считать, что, во-первых,

$$
F(0, \varepsilon) \equiv 0, \quad F_{u}^{\prime}(0, \varepsilon) \equiv 0, \quad F(u, 0)=F_{2}(u, u)+F_{3}(u, u, u)+\cdots,
$$

где $F_{2}, F_{3}, \ldots$ - квадратичная, кубическая и т.д. симметричные формы; во-вторых, справедливо неравенство

$$
\operatorname{Re} d<0
$$

где

$$
\begin{aligned}
& d=2\left(F_{2}\left(c_{0}, a\right), b\right)+2\left(F_{2}\left(c_{2}, \bar{a}\right), b\right)+3\left(F_{3}(a, a, \bar{a}), b\right), \\
& c_{0}=-2 A_{0}^{-1} F_{2}(a, \bar{a}), \quad c_{2}=\left[2 i \omega_{0} I-A_{0}\right]^{-1} F_{2}(a, a),
\end{aligned}
$$

$I$ - единичная матрица. Заметим, что (1.5) - первая ляпуновская величина обыкновенного дифференциального уравнения, получающегося из (1.1) при $\varepsilon=0$ и при отбрасывании граничных условий.

Заключительная группа ограничений относится к матрицам $A_{0}-z D$ и $-D$, где параметр $z$ пробегает полуось $(0,+\infty)$. Считаем, что обе они являются гурвицевыми, причем собственные значения $\lambda(z), \bar{\lambda}(z)$ первой из них, обращающиеся при $z=0$ в $\pm i \omega_{0}$, при увеличении параметра $z$ отходят от мнимой оси общим образом, т.е.

$$
\operatorname{Re}(D a, b)>0 \text {. }
$$

Как известно (см., например, [1], [2]), при условиях (1.2), (1.4) отвечающая краевой задаче (1.1) точечная модель, т.е. система обыкновенных дифференциальных уравнений

$$
\dot{u}=A(\varepsilon) u+F(u, \varepsilon),
$$

при всех достаточно малых $\varepsilon>0$ имеет экспоненциально орбитально устойчивый цикл

$$
\begin{aligned}
u=u_{*}(\tau, \varepsilon): \quad u_{*} & =\sqrt{\varepsilon} u_{1}(\tau)+\varepsilon u_{2}(\tau)+\varepsilon^{3 / 2} u_{3}(\tau, \varepsilon), \\
\frac{d \tau}{d t} & =1+\varepsilon \alpha_{0}+\varepsilon^{2} \alpha_{1}(\varepsilon),
\end{aligned}
$$

возникающий в результате классической бифуркации Андронова-Хопфа. Здесь функции $u_{1}(\tau), u_{2}(\tau)$ и постоянная $\alpha_{0}$ задаются равенствами

$$
\begin{aligned}
& u_{1}(\tau)=\xi_{0}\left[a \exp \left(i \omega_{0} \tau\right)+\bar{a} \exp \left(-i \omega_{0} \tau\right)\right], \\
& u_{2}(\tau)=\xi_{0}^{2}\left[c_{0}+c_{2} \exp \left(2 i \omega_{0} \tau\right)+\bar{c}_{2} \exp \left(-2 i \omega_{0} \tau\right)\right], \\
& \xi_{0}=\sqrt{-\frac{\phi_{0}}{\operatorname{Re} d}}, \quad \omega_{0} \alpha_{0}=\operatorname{Im}\left(A_{1} a, b\right)+\operatorname{Im} d \cdot \xi_{0}^{2},
\end{aligned}
$$


в которых $d$ - величина (1.5), $c_{0}, c_{2}$ - векторы (1.6). Далее, фигурирующая в (1.9) $2 \pi / \omega_{0}$-периодическая по $\tau$ вектор-функция $u_{3}(\tau, \varepsilon)$ ограничена равномерно по $\tau, \varepsilon$ вместе с любым фиксированным числом своих производных по $\tau$, а $\alpha_{1}(\varepsilon)$ - некоторая достаточно гладкая по $\varepsilon \in\left[0, \varepsilon_{0}\right]$ скалярная функция.

Нетрудно заметить, что цикл $(1.9),(1.10)$ является в то же время пространственно однородным (не зависящим от пространственной переменной $x$ ) решением исходной краевой задачи (1.1). Однако из факта устойчивости этого цикла в рамках системы (1.8), вообе говоря, не следует его устойчивость в распределенной модели (1.1). Действительно, пусть у матрицы $A_{0}-z D$ при некотором $z=z_{0}>0$ существует собственное значение в полуплоскости $\operatorname{Re} \lambda>0$. Тогда нулевое решение краевой задачи (1.1), а значит, и все бифурцирующие из него автоколебания, как однородные, так и нетривиально зависящие от $x$, оказываются неустойчивыми с показателем экспоненты порядка единицы. Таким образом, в данном случае интересующая нас проблема отыскания у системы (1.1) устойчивых автоволн малой амплитуды теряет смысл. В связи с этим становится понятной роль наложенных выше ограничений на матрицу $A_{0}-z D, z>0$ : в совокупности они исключают описанную ситуацию.

Итак, естественным образом возникает вопрос об устойчивости и бифуркациях однородного цикла (1.9) в фазовом пространстве $W_{2}^{1}\left([0, \pi] ; \mathbb{R}^{N}\right)$ задачи (1.1). Как будет установлено ниже, при надлежащей деформации матричного коэффициента диффузии $D$ упомянутый цикл теряет устойчивость и порождает два устойчивых пространственно неоднородных периодических режима, т.е. происходит бифуркация типа вилки.

1.2. Метод квазинормальных форм. Структура однородного цикла (1.9) наводит на мысль, что и возможные бифурцирующие из него пространственно неоднородные режимы краевой задачи (1.1) устроены аналогично, т.е. должны иметь амплитуды порядка $\sqrt{\varepsilon}$. Во всяком случае, ниже мы ограничимся анализом именно таких автоколебаний и для их отыскания воспользуемся так называемым методом квазинормальных форм, предложенным Ю. С. Колесовым в начале 1980-х годов. Не останавливаясь на истории вопроса, отметим лишь монографии [3]-[6], в которых содержатся как подробное изложение данного метода, так и различные примеры его применения.

В алгоритмическом плане метод квазинормальных форм состоит в следующем. Подставим в (1.1) формальный асимптотический ряд по целым степеням $\sqrt{\varepsilon}$ :

$$
u=\sqrt{\varepsilon} u_{1}(t, s, x)+\varepsilon u_{2}(t, s, x)+\varepsilon^{3 / 2} u_{3}(t, s, x)+\cdots,
$$

в котором $s=\varepsilon t$,

$$
u_{1}=\xi(s, x) a \exp \left(i \omega_{0} t\right)+\bar{\xi}(s, x) \bar{a} \exp \left(-i \omega_{0} t\right)
$$

$\xi(s, x)$ - неизвестная комплексная амплитуда колебаний, а функции $u_{2}(t, s, x)$, $u_{3}(t, s, x)$ и т.д. периодичны по $t$ с периодом $2 \pi / \omega_{0}$. Приравнивая затем коэффициенты при $\varepsilon$, для определения $u_{2}$ с учетом свойств (1.3) нелинейности 
$F(u, \varepsilon)$ приходим к уравнению

$$
\frac{\partial u_{2}}{\partial t}-A_{0} u_{2}=F_{2}\left(u_{1}, u_{1}\right)
$$

в котором переменные $s, x$ будем рассматривать как параметры. Несложный подсчет показывает, что это уравнение допускает периодическое решение

$$
u_{2}=|\xi|^{2} c_{0}+\xi^{2} c_{2} \exp \left(2 i \omega_{0} t\right)+\bar{\xi}^{2} \bar{c}_{2} \exp \left(-2 i \omega_{0} t\right)
$$

где $c_{0}, c_{2}-$ векторы (1.6).

После приравнивания коэффициентов при $\varepsilon^{3 / 2}$ для $u_{3}$ получаем аналогичное (1.13) уравнение

$$
\frac{\partial u_{3}}{\partial t}-A_{0} u_{3}=-\frac{\partial u_{1}}{\partial s}+D \frac{\partial^{2} u_{1}}{\partial x^{2}}+A_{1} u_{1}+2 F_{2}\left(u_{1}, u_{2}\right)+F_{3}\left(u_{1}, u_{1}, u_{1}\right),
$$

где, напомним, $A_{1}$ - матрица, фигурирующая в определении $\phi_{0}$ (см. (1.2)). Так как нас интересует периодическое по $t$ решение этого уравнения, то мы должны приравнять к нулю скалярное произведение коэффициента его правой части при $\exp \left(i \omega_{0} t\right)$ на вектор $b$. Указанные действия с учетом равенств $(1.12),(1.14)$ приводят к уравнению

$$
\frac{\partial \xi}{\partial s}=(D a, b) \frac{\partial^{2} \xi}{\partial x^{2}}+\left(A_{1} a, b\right) \xi+d|\xi|^{2} \xi
$$

где $d$ - величина (1.5), а $\bar{\xi}$ удовлетворяет комплексно сопряженному уравнению. В соответствии с (1.1) уравнение (1.15), которое в силу условия (1.7) является параболическим, следует дополнить граничными условиями

$$
\left.\frac{\partial \xi}{\partial x}\right|_{x=0}=\left.\frac{\partial \xi}{\partial x}\right|_{x=\pi}=0
$$

Получившуюся краевую задачу (1.15), (1.16) будем называть квазинормальной формой исходной задачи (1.1). Подобное название оправдано тем, что при $D=0$ уравнение (1.15) представляет собой укороченную нормальную форму соответствующего обыкновенного уравнения (1.8) на устойчивом двумерном локальном инвариантном многообразии. Если же $D \neq 0$, то при выводе задачи $(1.15),(1.16)$ отбрасываются слагаемые, формально малые по порядку относительно $\varepsilon$, но по силе не являющиеся подчиненными остальным. Однако несмотря на это некоторые утверждения о соответствии между стационарными режимами краевых задач (1.15), (1.16) и (1.1) все же имеют место. Для их формулировки нам потребуются следующие два определения.

ОПРЕДЕЛЕНИЕ 1.1. Автомодельным ииклом краевой задачи (1.15), (1.16) назовем ее периодическое по $s$ решение вида

$$
\xi=\xi_{0}(x) \exp \left(i \alpha_{0} s\right)
$$

где $\alpha_{0} \in \mathbb{R}$ - некоторая постоянная. 
ОПРЕДЕЛЕНИЕ 1.2. Двумерным автомоделъным тором задачи (1.15), (1.16) будем называть ее квазипериодическое решение вида

$$
\xi=\xi_{0}(s, x) \exp \left(i \alpha_{0} s\right), \quad \alpha_{0}=\text { const } \in \mathbb{R},
$$

где комплекснозначная функция $\xi_{0}(s, x)$ периодична по $s$ с некоторым периодом $T_{0}>0$. Предполагаем, что решение (1.18) не приводится к виду (1.17).

Справедливо следующее утверждение [3], [6], представляющее собой аналог известных теорем Боголюбова об усреднении на бесконечном промежутке времени.

ТЕорема 1.1. Предположим, что квазинормальная форма (1.15), (1.16) имеет автомодельный цикл (двумерный тор), экспоненииально орбитально устойчивый или дихотомичный в метрике фбазового пространства $(\operatorname{Re} \xi, \operatorname{Im} \xi) \in W_{2}^{1}(0, \pi) \times W_{2}^{1}(0, \pi)$. Тогда найдется такое достаточно малое $\varepsilon_{0}>0$, что при всех $0<\varepsilon \leqslant \varepsilon_{0}$ в исходной краевой задаче (1.1) ему соответствует иикл (двумерный инвариантный тор) с теми же свойствами устойчивости и с асимптотикой (1.11), (1.12), (1.17) (или (1.18)).

1.3. Локальный анализ квазинормальной формы. Дальнейший анализ как квазинормальной формы (1.15), (1.16), так и исходной системы (1.1) проведем при двух дополнительных ограничениях. Во-первых, предположим, что диффузия $D$ в (1.1) пропорциональна некоторому вспомогательному параметру $\nu>0$, т.е.

$$
D=\nu D_{0}
$$

Здесь матрица $D_{0}$ фиксирована, причем $\operatorname{Re}\left(D_{0} a, b\right)=1$, а матрицы $A_{0}-z D_{0}$ при любых $z>0$ и $-D_{0}$ являются гурвицевыми. Менять же в последующем будем только параметр $\nu$. Во-вторых, считаем выполненным неравенство

$$
x_{0} y_{0}>1
$$

где

$$
x_{0}=-\operatorname{Im}\left(D_{0} a, b\right), \quad y_{0}=\frac{\operatorname{Im} d}{\operatorname{Re} d} .
$$

Сформулированная выше теорема 1.1 о соответствии позволяет уже достаточно просто решить вопрос об устойчивости однородного цикла (1.9) в рамках распределенной модели (1.1). Действительно, упомянутому циклу в квазинормальной форме (1.15), (1.16) отвечает автомодельный цикл

$$
\xi=\xi_{0} \exp \left(i \omega_{0} \alpha_{0} s\right)
$$

где постоянные $\xi_{0}, \alpha_{0}$ определены в (1.10). Выполним, далее, в $(1.15),(1.16)$ замены

$$
\xi=\xi_{0}(1+h) \exp \left(i \omega_{0} \alpha_{0} s\right), \quad \bar{\xi}=\xi_{0}(1+\bar{h}) \exp \left(-i \omega_{0} \alpha_{0} s\right)
$$


и отбросим нелинейные по $h$ слагаемые. В результате убеждаемся, что устойчивость цикла (1.22) определяется по расположению характеристических показателей линейной краевой задачи, которая с учетом равенств (1.19), (1.21) принимает вид

$$
\frac{\partial h}{\partial s}=\nu\left(1-i x_{0}\right) \frac{\partial^{2} h}{\partial x^{2}}-\phi_{0}\left(1+i y_{0}\right)(h+\bar{h}),\left.\quad \frac{\partial h}{\partial x}\right|_{x=0}=\left.\frac{\partial h}{\partial x}\right|_{x=\pi}=0
$$

Наконец, применяя к (1.23) метод Фурье по системе функций $\cos k x, k=0,1, \ldots$, приходим к выводу, что интересующие нас характеристические показатели это спектр семейства матриц

$$
\begin{gathered}
C_{k}(\nu)=-\left(\begin{array}{cc}
\phi_{0}\left(1+i y_{0}\right) & \phi_{0}\left(1+i y_{0}\right) \\
\phi_{0}\left(1-i y_{0}\right) & \phi_{0}\left(1-i y_{0}\right)
\end{array}\right)-\left(\begin{array}{cc}
k^{2} \nu\left(1-i x_{0}\right) & 0 \\
0 & k^{2} \nu\left(1+i x_{0}\right)
\end{array}\right) \\
k=0,1, \ldots
\end{gathered}
$$

Заметим, что матрица (1.24) при $k=0$ имеет одно нулевое собственное значение (что естественно, так как (1.23) - линеаризация на цикле), а второе ее собственное значение отрицательно. Что же касается матриц $C_{k}(\nu), k \geqslant 1$, то в силу условия (1.20) все они являются гурвицевыми при

$$
\nu>\nu_{*}, \quad \text { где } \nu_{*}=\frac{2 \phi_{0}\left(x_{0} y_{0}-1\right)}{1+x_{0}^{2}}>0,
$$

а при выполнении строго противоположного (1.25) неравенства положительное собственное значение существует, например, у матрицы $C_{1}(\nu)$. В критическом же случае $\nu=\nu_{*}$ у матрицы $C_{1}(\nu)$ появляется нулевое собственное значение, а матрицы $C_{k}(\nu), k \geqslant 2$, по-прежнему гурвицевы.

Из проделанных построений следует, что при фиксированном $\nu>\nu_{*}\left(<\nu_{*}\right)$ однородный цикл (1.22) квазинормальной формы (1.15), (1.16) экспоненциально орбитально устойчив (неустойчив), а значит, аналогичными свойствами устойчивости обладает при всех достаточно малых $\varepsilon>0$ и цикл (1.9) исходной задачи (1.1). Таким образом, остается разобраться с характером бифуркаций, происходящих с этими циклами при прохождении параметра $\nu$ через критическое значение $\nu_{*}$. Для однородного цикла (1.22) соответствующий бифуркационный анализ проводится в данном пункте.

Подставим в уравнение (1.15) соотношения

$$
(D a, b)=\nu\left(1-i x_{0}\right), \quad \nu=\nu_{*}-\mu, \quad 0<\mu \ll 1,
$$

где $\mu$ - новый малый параметр, характеризующий отклонение величины $\nu$ от введенного выше критического значения $\nu_{*}$. При выполнении данных условий будем интересоваться существованием и устойчивостью у квазинормальной формы (1.15), (1.16) пространственно неоднородных автомодельных циклов вида

$$
\xi=\xi_{0}(1+h(x, \mu)) \exp \left[i\left(\omega_{0} \alpha_{0}+\alpha_{*}(\mu)\right) s\right]
$$

где $h(x, 0) \equiv 0, \alpha_{*}(0)=0$. 
Учитывая равенства (1.26), (1.27) и (1.15), (1.16), для отыскания комплекснозначной функции $h(x, \mu)$ и вещественной поправки к частоте $\alpha_{*}(\mu)$ приходим к краевой задаче

$$
\begin{gathered}
i \alpha_{*}(\mu)(1+h)=\left(\nu_{*}-\mu\right)\left(1-i x_{0}\right) h^{\prime \prime}-\phi_{0}\left(1+i y_{0}\right)\left[h+\bar{h}+2|h|^{2}+h^{2}+|h|^{2} h\right], \\
\left.h^{\prime}\right|_{x=0}=\left.h^{\prime}\right|_{x=\pi}=0
\end{gathered}
$$

где $h^{\prime}, h^{\prime \prime}-$ производные по $x$. Заметим, что в силу равенств $\operatorname{det} C_{k}\left(\nu_{*}\right)=0$, $k=0,1$, получающаяся из (1.28) при $\alpha_{*}=\mu=0$ после отбрасывания нелинейных слагаемых краевая задача

$$
\Pi h \equiv \nu_{*}\left(1-i x_{0}\right) h^{\prime \prime}-\phi_{0}\left(1+i y_{0}\right)[h+\bar{h}]=0,\left.\quad h^{\prime}\right|_{x=0}=\left.h^{\prime}\right|_{x=\pi}=0
$$

имеет два линейно независимых нетривиальных решения:

$$
h_{1}(x)=\left(\gamma_{1}+i \gamma_{2}\right) \cos x, \quad h_{2}(x)=i,
$$

где $\gamma_{1}=1-x_{0} y_{0}, \gamma_{2}=x_{0}+y_{0}$. Поэтому для построения асимптотики пары функций $\alpha_{*}(\mu), h(x, \mu)$, обращающих (1.28) в тождество, воспользуемся некоторым вариантом одночастотного метода из [7]. В данном случае, обыгрывая существование у краевой задачи (1.29) первого из решений (1.30), будем искать функции $\alpha_{*}(\mu), h(x, \mu)$ в виде рядов по целым степеням $\mu$ и $\sqrt{\mu}$ соответственно:

$$
\begin{gathered}
\alpha_{*}(\mu)=\alpha_{1, *} \mu+\alpha_{2, *} \mu^{2}+\cdots, \\
h=\sqrt{\mu} h_{0}(x)+\mu h_{1}(x)+\mu^{3 / 2} h_{2}(x)+\cdots, \quad h_{0}(x)=\eta_{0}\left(\gamma_{1}+i \gamma_{2}\right) \cos x,
\end{gathered}
$$

где $\eta_{0}$ - произвольная (подлежащая определению) вещественная амплитуда.

Подставляя ряды (1.31), (1.32) в (1.28) и приравнивая коэффициенты при одинаковых степенях $\mu$, для функций $h_{k}, k \geqslant 1$, получаем рекуррентную последовательность линейных неоднородных краевых задач вида

$$
\Pi h_{k}=f_{k}(x),\left.\quad h_{k}^{\prime}\right|_{x=0}=\left.h_{k}^{\prime}\right|_{x=\pi}=0 .
$$

Неизвестные же коэффициенты ряда (1.31) вместе с амплитудой $\eta_{0}$ (и другими возникающими в ходе алгоритма амплитудами) определяются из условий разрешимости данных краевых задач, которые имеют вид

$$
\operatorname{Im}\left[\left(1-i y_{0}\right) \int_{0}^{\pi} f_{k}(x) d x\right]=0, \quad \operatorname{Re}\left[\left(1+i x_{0}\right) \int_{0}^{\pi} f_{k}(x) \cos x d x\right]=0 .
$$

На первом шаге алгоритма рассмотрению подлежит краевая задача (1.33) при $k=1$, в которой

$$
f_{1}(x)=i \alpha_{1, *}+\phi_{0}\left(1+i y_{0}\right) \eta_{0}^{2}\left(3 \gamma_{1}^{2}+\gamma_{2}^{2}+2 i \gamma_{1} \gamma_{2}\right) \cos ^{2} x .
$$

Далее, поскольку неоднородность (1.35) представляет собой линейную комбинацию функций $1, \cos 2 x$, то второе условие разрешимости (1.34) здесь выполняется автоматически. Первое же из них приводит к равенству

$$
\alpha_{1, *}=-\phi_{0}\left(1+y_{0}^{2}\right) \eta_{0}^{2} \gamma_{1} \gamma_{2}
$$


И наконец, после нахождения $\alpha_{1, *}$ решение $h_{1}(x)$ рассматриваемой краевой задачи, удовлетворяющее дополнительным условиям

$$
\operatorname{Im}\left[\left(1-i y_{0}\right) \int_{0}^{\pi} h_{1}(x) d x\right]=0, \quad \operatorname{Re}\left[\left(1+i x_{0}\right) \int_{0}^{\pi} h_{1}(x) \cos x d x\right]=0,
$$

определяется однозначно и имеет вид

$$
\begin{gathered}
h_{1}(x)=\eta_{0}^{2} h_{1,0}(x), \quad h_{1,0}(x)=\left(1+i y_{0}\right) R_{0}+\left(R_{1}+i R_{2}\right) \cos 2 x, \\
R_{0}=\frac{1}{4}\left(2 y_{0} \gamma_{1} \gamma_{2}-3 \gamma_{1}^{2}-\gamma_{2}^{2}\right) \\
R_{1}=\frac{1}{12}\left(3 \gamma_{1}^{2}-\gamma_{2}^{2}\right), \quad R_{2}=\frac{1}{24} \frac{\gamma_{2}}{\gamma_{1}}\left(9 \gamma_{1}^{2}+\gamma_{2}^{2}\right) .
\end{gathered}
$$

На втором шаге приходим к краевой задаче (1.33) при $k=2$ с неоднородностью

$$
f_{2}=i \alpha_{1, *} h_{0}+\left(1-i x_{0}\right) h_{0}^{\prime \prime}+\phi_{0}\left(1+i y_{0}\right)\left[4 \operatorname{Re}\left(h_{0} \bar{h}_{1}\right)+2 h_{0} h_{1}+\left|h_{0}\right|^{2} h_{0}\right],
$$

которая, как следует из явного вида $h_{0}, h_{1}$ (см. (1.32), (1.38)), представляет собой линейную комбинацию нечетных гармоник $\cos x, \cos 3 x$. Поэтому в данном случае автоматически выполняется первое из условий разрешимости (1.34). Второе же из этих условий с учетом формул (1.36) и (1.38) после некоторых преобразований приобретает вид

$$
\left(1+x_{0}^{2}\right)\left(x_{0} y_{0}-1\right) \eta_{0}+\phi_{0} Q\left(x_{0}, y_{0}\right) \eta_{0}^{3}=0,
$$

где

$$
Q\left(x_{0}, y_{0}\right)=-3 \gamma_{1}^{4}-\frac{1}{6} \gamma_{2}^{4}+\frac{3}{2} \gamma_{1}^{2} \gamma_{2}^{2}+2 y_{0} \gamma_{1}^{3} \gamma_{2}
$$

Будем рассматривать равенство (1.39) как уравнение для отыскания неизвестной амплитуды $\eta_{0}$. В связи с этим обратим внимание на то, что величина (1.40) является отрицательной при всех $x_{0}, y_{0}$, удовлетворяющих условию (1.20). Действительно, в силу четности $Q$ (где $\left.Q\left(-x_{0},-y_{0}\right) \equiv Q\left(x_{0}, y_{0}\right)\right)$ достаточно ограничиться областью

$$
\left\{\left(x_{0}, y_{0}\right): x_{0} y_{0}>1, x_{0}>0\right\} .
$$

Далее, заметим, что область (1.41) расслаивается на гиперболы вида

$$
\left\{\left(x_{0}, y_{0}\right): y_{0}=\frac{\sqrt{p} x_{0}+1}{x_{0}-\sqrt{p}}, x_{0}>\sqrt{p}\right\},
$$

где $p>0$. На каждой из таких кривых имеем

$$
Q=\gamma_{2}^{4}\left(-3 p^{2}-\frac{1}{6}+\frac{3}{2} p-2 y_{0} p \sqrt{p}\right)<\gamma_{2}^{4}\left(-5 p^{2}+\frac{3}{2} p-\frac{1}{6}\right)<0,
$$

так как в этом случае $\gamma_{1}=-\sqrt{p} \gamma_{2}$ и $y_{0}>\sqrt{p}$.

Установленные свойства функции (1.40) позволяют сначала определить из (1.39) требуемую амплитуду $\eta_{0}$ посредством равенства

$$
\eta_{0}=\sqrt{\frac{\left(1+x_{0}^{2}\right)\left(1-x_{0} y_{0}\right)}{\phi_{0} Q}}
$$


а затем и функцию $h_{2}=h_{2}^{0}+\eta_{1}\left(\gamma_{1}+i \gamma_{2}\right) \cos x$, где $h_{2}^{0}$ - единственное решение краевой задачи (1.33) при $k=2$, удовлетворяющее аналогичным (1.37) условиям нормировки, $\eta_{1}-$ произвольная вещественная постоянная.

Как нетрудно убедиться, после выбора решения (1.42) уравнения разветвления (1.39) приведенный алгоритм продолжается неограниченно.

В самом деле, в общем случае на шаге с номером $k=2 j-1, j=1,2, \ldots$, неоднородность в краевой задаче (1.33) имеет вид $f_{2 j-1}=i \alpha_{j, *}+f_{2 j-1}^{0}(x)$, где $f_{2 j-1}^{0}$ представляет собой линейную комбинацию гармоник $\cos 2 n x, n=0,1, \ldots, j$, с коэффициентами, зависящими от $\alpha_{m, *}, m \leqslant j-1$, и $\eta_{r}, r \leqslant j$. Таким образом, второе из условий разрешимости (1.34) здесь выполняется автоматически. Первое же из них позволяет найти очередную постоянную $\alpha_{j, *}$. После этого функция $h_{2 j-1}(x)$, удовлетворяющая равенствам вида (1.37), определяется уже однозначно.

На шаге с номером $k=2 j, j=1,2, \ldots$, ситуация несколько иная: неоднородность $f_{2 j}$ является линейной комбинацией нечетных гармоник $\cos (2 n+1) x$, $n=0, \ldots, j$. Поэтому в данном случае автоматически выполняется первое из условий разрешимости (1.34). Выполнения же второго добиваемся за счет слагаемого $\eta_{j-1}\left(\gamma_{1}+i \gamma_{2}\right) \cos x$, с точностью до которого определяется $h_{2(j-1)}$. Заметим еще, что, как это обычно происходит в теории ветвления, для отыскания $\eta_{j-1}, j \geqslant 2$, в отличие от (1.39) получаются уже линейные неоднородные уравнения вида $2 \phi_{0} Q \eta_{0}^{2} \eta_{j-1}=\psi_{j}$.

После нахождения $\eta_{j-1}$ функция $h_{2 j}$ определяется в виде

$$
h_{2 j}=h_{2 j}^{0}(x)+\eta_{j}\left(\gamma_{1}+i \gamma_{2}\right) \cos x,
$$

где $h_{2 j}^{0}(x)$ удовлетворяет аналогичным (1.37) условиям нормировки, а $\eta_{j}-$ произвольная вещественная постоянная. Далее переходим к шагу с номером $2 j+1$, и весь описанный процесс повторяется.

Строгий смысл изложенным формальным построениям придает следующее утверждение.

ТеОрема 1.2. Пусть выполнены условия (1.20) и (1.26). Тогда найдется такое достаточно малое $\mu_{0}>0$, что при всех $0<\mu \leqslant \mu_{0}$ существуют две пары функций

$$
\left(\alpha_{*}(\mu), h_{ \pm}(x, \mu)\right): \quad h_{-}(x, \mu)=h_{+}(\pi-x, \mu),
$$

обращающие краевую задачу (1.28) в тождество. При этом функиии $\alpha_{*}(\mu)$, $h_{+}(x, \mu)$ допускают асимптотику (1.31), (1.32), (1.42), а соответствующие парам (1.43) автомодельные чиклы (1.27) краевой задачи (1.15), (1.16) экспоненциально орбитально устойчивы.

ДокАзАтельство. Сформулированная теорема представляет собой автоколебательный вариант классической бифуркационной теоремы Тьюринга-Пригожина [8]. Ее обоснование существенно опирается на тот факт, что при условиях (1.26) и при всех достаточно малых по модулю значениях $\mu$ краевая задача $(1.15),(1.16)$ в окрестности однородного цикла (1.22) имеет экспоненциально 
орбитально устойчивое двумерное интегральное многообразие [9], достаточно гладко зависящее от параметра $\mu$.

Обратимся теперь непосредственно к доказательству теоремы и заметим, что в силу специфики задачи (1.15), (1.16) упомянутое выше многообразие обладает некоторыми дополнительными свойствами. Для того чтобы убедиться в этом, выполним в (1.15), (1.16) при условиях (1.26) замену

$$
\xi=\xi_{0}[1+h(s, x)] \exp (i \psi(s)),
$$

где

$$
\operatorname{Im}\left[\left(1-i y_{0}\right) \int_{0}^{\pi} h(s, x) d x\right]=0
$$

$\xi_{0}$ - амплитуда из (1.22). В результате для комплекснозначной функции $h(s, x)$ и вещественной фазовой переменной $\psi(s)$ получаем систему треугольного вида

$$
\begin{gathered}
\frac{\partial h}{\partial s}=\left(\nu_{*}-\mu\right)\left(1-i x_{0}\right) \frac{\partial^{2} h}{\partial x^{2}}+G_{1}(h)-i(1+h) G_{2}(h),\left.\quad \frac{\partial h}{\partial x}\right|_{x=0}=\left.\frac{\partial h}{\partial x}\right|_{x=\pi}=0, \\
\frac{d \psi}{d s}=\omega_{0} \alpha_{0}+G_{2}(h),
\end{gathered}
$$

в которой $\omega_{0} \alpha_{0}$ - частота цикла (1.22),

$$
G_{1}=-\phi_{0}\left(1+i y_{0}\right)\left[h+\bar{h}+2|h|^{2}+h^{2}+|h|^{2} h\right], \quad G_{2}=\frac{\operatorname{Im}\left[\left(1-i y_{0}\right) l\left(G_{1}(h)\right)\right]}{1+\operatorname{Im}\left[\left(i+y_{0}\right) l(h)\right]},
$$

$l(*)$ - среднее значение по $x$. Поэтому интересующее нас многообразие задается аналогичным (1.44) равенством

$$
\xi=\xi_{0}\left[1+\eta\left(\gamma_{1}+i \gamma_{2}\right) \cos x+\eta^{2} h_{1,0}(x)+H(\eta, x, \mu)\right] \exp (i \psi),
$$

а система уравнений на нем также имеет треугольный вид:

$$
\begin{aligned}
\frac{d \eta}{d s} & =\frac{\left(1+x_{0}^{2}\right)\left(x_{0} y_{0}-1\right)}{\gamma_{2} x_{0}-\gamma_{1}} \mu \eta+\frac{\phi_{0} Q\left(x_{0}, y_{0}\right)}{\gamma_{2} x_{0}-\gamma_{1}} \eta^{3}+\Phi_{1}(\eta, \mu), \\
\frac{d \psi}{d s} & =\omega_{0} \alpha_{0}-\phi_{0}\left(1+y_{0}^{2}\right) \gamma_{1} \gamma_{2} \eta^{2}+\Phi_{2}(\eta, \mu) .
\end{aligned}
$$

Здесь $h_{1,0}(x)$ - функция из $(1.38),(\eta, \psi)$ - вещественные параметры на многообразии $(1.45)$, причем $\psi \in[0,2 \pi](\bmod 2 \pi), \eta \in[-q, q]$, где $q>0$ достаточно мало, а комплекснозначная функция $H$ и вещественные функции $\Phi_{j}$, $j=1,2$, достаточно гладко зависят от своих переменных и обладают следующими свойствами:

$$
\begin{aligned}
& H(0, x, \mu) \equiv \frac{\partial H}{\partial \eta}(0, x, 0) \equiv \frac{\partial^{2} H}{\partial \eta^{2}}(0, x, 0) \equiv 0,\left.\quad \frac{\partial H}{\partial x}\right|_{x=0, \pi}=0 \\
& \operatorname{Im}\left[\left(1-i y_{0}\right) \int_{0}^{\pi} H(\eta, x, \mu) d x\right] \equiv 0 \\
& \Phi_{1}(0, \mu) \equiv 0, \quad \frac{\partial \Phi_{1}}{\partial \eta}(0,0)=\frac{\partial^{2} \Phi_{1}}{\partial \eta^{2}}(0,0)=\frac{\partial^{3} \Phi_{1}}{\partial \eta^{3}}(0,0)=\frac{\partial^{2} \Phi_{1}}{\partial \eta \partial \mu}(0,0)=0 \\
& \Phi_{2}(0, \mu) \equiv 0, \quad \frac{\partial \Phi_{2}}{\partial \eta}(0,0)=\frac{\partial^{2} \Phi_{2}}{\partial \eta^{2}}(0,0)=0 .
\end{aligned}
$$


Из перечисленных свойств функций $\Phi_{j}, j=1,2$, вытекает, что после выполнения в системе (1.46) нормировки $\eta / \sqrt{\mu} \rightarrow \eta$ и отбрасывания слагаемых порядка $\mu^{3 / 2}$ и выше "укороченная" нормальная форма на многообразии (1.45) примет вид

$$
\begin{aligned}
\frac{d \eta}{d s} & =\mu\left[\frac{\left(1+x_{0}^{2}\right)\left(x_{0} y_{0}-1\right)}{\gamma_{2} x_{0}-\gamma_{1}} \eta+\frac{\phi_{0} Q\left(x_{0}, y_{0}\right)}{\gamma_{2} x_{0}-\gamma_{1}} \eta^{3}\right], \\
\frac{d \psi}{d s} & =\omega_{0} \alpha_{0}-\mu \phi_{0}\left(1+y_{0}^{2}\right) \gamma_{1} \gamma_{2} \eta^{2} .
\end{aligned}
$$

Отметим еще, что здесь справедливо стандартное утверждение о соответствии между грубыми стационарными режимами исходной краевой задачи (1.15), (1.16) и системы (1.47). Поэтому двум экспоненциально орбитально устойчивым циклам последней вида

$$
\eta= \pm \eta_{0}, \quad \frac{d \psi}{d s}=\omega_{0} \alpha_{0}-\mu \phi_{0}\left(1+y_{0}^{2}\right) \gamma_{1} \gamma_{2} \eta_{0}^{2},
$$

где $\eta_{0}$ - амплитуда (1.42), отвечают два устойчивых цикла задачи (1.15), (1.16) вида (1.27), переходящих друг в друга при замене $\pi-x \rightarrow x$. Остается добавить, что асимптотические представления (1.31), (1.32) - следствие гладкости многообразия (1.45) по $\mu$ (более того, используя развитые в монографии [10] конструкции, можно показать сходимость рядов (1.31), (1.32) при всех достаточно малых $\mu>0)$. Теорема доказана.

Просуммируем полученные результаты. Из проделанного выше анализа следует, что в рамках квазинормальной формы (1.15), (1.16) при условиях (1.19), (1.20) и при прохождении параметра $\nu$ через критическое значение $\nu_{*}$ наблюдается бифуркация типа вилки: однородный цикл (1.22) устойчив при $\nu>\nu_{*}$, а при $0<\nu_{*}-\nu \ll 1$ теряет ее и порождает два устойчивых пространственно неоднородных цикла вида (1.27). Что же касается исходной краевой задачи (1.1), то для нее, опираясь на теорему 1.1, мы можем утверждать пока лишь следующее.

ТЕОРема 1.3. По любым достаточно малым $\mu_{2}>\mu_{1}>0$ найдется такое $\varepsilon_{0}=\varepsilon_{0}\left(\mu_{1}, \mu_{2}\right)>0$, что при дополнительных условиях (1.19), (1.20), (1.26) и при всех $\mu_{1} \leqslant \mu \leqslant \mu_{2}, 0<\varepsilon \leqslant \varepsilon_{0}$ краевая задача (1.1) имеет два устойчивых пространственно неоднородных иикла, отвечающих автомодельным ииклам (1.27) квазинормальной формы (1.15), (1.16).

Проблема здесь заключается в том, что, находясь в рамках применимости теоремы 1.1, мы вынуждены отделять параметр $\mu$ от нуля для обеспечения требуемой грубости автомодельных циклов (1.27). А это значит, что теорема 1.3 не является бифуркационной в строгом смысле данного слова, так как пока мы не можем менять параметры $\varepsilon$ и $\mu$ независимо друг от друга. Вопрос же о том, что происходит в исходной задаче (1.1) при независимом изменении $\varepsilon$ и $\mu$, на протяжении более чем двадцати лет (начиная с работы [11]) оставался открытым. Точнее говоря, в [11] был получен аналог теоремы 1.3 в случае $N=2$ и сформулирована гипотеза (принадлежащая Ю. С. Колесову) о том, что 
в условиях этой теоремы при фиксированном $\varepsilon>0$ и при $\mu \rightarrow 0$ в окрестности однородного цикла происходит бесконечно много бифуркаций, каждая из которых уменьшает "степень" зависимости от $x$ пространственно неоднородных стационарных режимов.

Решению поставленной проблемы и посвящена настоящая статья. А именно, ниже устанавливается, что упомянутая гипотеза не соответствует действительности. На самом деле в системе (1.1) при условиях (1.19), (1.20) реализуется такая же ситуация, как и в случае квазинормальной формы (1.15), (1.16): при прохождении параметра $\nu$ через некоторое критическое значение $\nu(\varepsilon), \nu(0)=\nu_{*}$, в ней наблюдается единственная бифуркация типа вилки.

\section{§2. Основной результат}

2.1. Уточнение постановки задачи. В первую очередь в уточнении нуждается упомянутое выше критическое значение $\nu(\varepsilon), \nu(0)=\nu_{*}$, параметра $\nu$, при котором в краевой задаче (1.1) и происходит ожидаемая бифуркация. Для нахождения этого значения сначала выполним в системе (1.1) при условии (1.19) замену времени $\tau=\sigma(\varepsilon) t$, где $\sigma(\varepsilon)=1+\varepsilon \alpha_{0}+\varepsilon^{2} \alpha_{1}(\varepsilon)$ (см. (1.9)), а затем линеаризуем получившуюся краевую задачу на однородном цикле $u=u_{*}(\tau, \varepsilon)$. В результате приходим к линейной краевой задаче

$$
\sigma(\varepsilon) \frac{\partial h}{\partial \tau}=\varepsilon \nu D_{0} \frac{\partial^{2} h}{\partial x^{2}}+A(\tau, \varepsilon) h,\left.\quad \frac{\partial h}{\partial x}\right|_{x=0}=\left.\frac{\partial h}{\partial x}\right|_{x=\pi}=0,
$$

где $A(\tau, \varepsilon)=A(\varepsilon)+\left.F_{u}^{\prime}(u, \varepsilon)\right|_{u=u_{*}(\tau, \varepsilon)}$. Заметим, далее, что из формул (1.9), (1.10) вытекает справедливое равномерно по $0 \leqslant \tau \leqslant 2 \pi / \omega_{0}$ асимптотическое представление

$$
A(\tau, \varepsilon)=A_{0}+\sqrt{\varepsilon} A_{0,1}(\tau)+\varepsilon A_{1}(\tau, \varepsilon), \quad A_{1}(\tau, \varepsilon)=A_{1}(\tau, 0)+O(\sqrt{\varepsilon}),
$$

в котором

$$
\begin{aligned}
& A_{0,1}(\tau) h=2 \xi_{0}(\left.F_{2}(a, h) \exp \left(i \omega_{0} \tau\right)+F_{2}(\bar{a}, h) \exp \left(-i \omega_{0} \tau\right)\right), \\
& A_{1}(\tau, 0) h=A_{1} h+\xi_{0}^{2}\left(2 F_{2}\left(c_{0}, h\right)+6 F_{3}(a, \bar{a}, h)\right) \\
&+\xi_{0}^{2}\left(2 F_{2}\left(c_{2}, h\right)+3 F_{3}(a, a, h)\right) \exp \left(2 i \omega_{0} \tau\right) \\
&+\xi_{0}^{2}\left(2 F_{2}\left(\bar{c}_{2}, h\right)+3 F_{3}(\bar{a}, \bar{a}, h)\right) \exp \left(-2 i \omega_{0} \tau\right) .
\end{aligned}
$$

Анализ краевой задачи (2.1) существенно облегчает то обстоятельство, что при подстановке в нее соотношения

$$
h=\sum_{k=0}^{\infty} h_{k}(\tau) \cos k x
$$

она "расслаивается" на счетную совокупность обыкновенных уравнений

$$
\sigma(\varepsilon) \frac{d h}{d \tau}=\left[A(\tau, \varepsilon)-\varepsilon k^{2} \nu D_{0}\right] h, \quad k=0,1, \ldots,
$$

для фигурирующих в (2.4) компонент $h=h_{k}(\tau)$. 
Зафиксируем произвольно номер $k$ и применим к соответствующей системе (2.5) предложенный в [12] алгоритм исследования устойчивости. Из близости ее коэффициентов к постоянным (см. (2.2), (2.3)) и из спектральных свойств матрицы $A_{0}$ заключаем, что при всех достаточно малых $\varepsilon>0$ рассматриваемая система имеет экспоненциально устойчивое (с показателем экспоненты порядка единицы) двумерное инвариантное подпространство решений, базисом в котором согласно результатам из [12] являются столбцы матрицы

$$
h=V_{k}(\tau, \varepsilon, \nu) \exp \left[\varepsilon \Lambda_{k}(\varepsilon, \nu) \tau\right] .
$$

Здесь $2 \pi / \omega_{0}$-периодическая по $\tau$ матрица-строка $V_{k}=\left[v_{k}(\tau, \varepsilon, \nu), \bar{v}_{k}(\tau, \varepsilon, \nu)\right]$ размера $N \times 2$ и квадратная матрица

$$
\Lambda_{k}(\varepsilon, \nu)=\left(\begin{array}{ll}
r_{1, k}(\varepsilon, \nu) & r_{2, k}(\varepsilon, \nu) \\
\bar{r}_{2, k}(\varepsilon, \nu) & \bar{r}_{1, k}(\varepsilon, \nu)
\end{array}\right)
$$

с комплексными элементами достаточно гладко зависят от параметров $\sqrt{\varepsilon}, \nu$ и раскладываются в асимптотические ряды по целым степеням $\sqrt{\varepsilon}$ и $\varepsilon$ :

$$
\begin{aligned}
v_{k}(\tau, \varepsilon, \nu) & =a \exp \left(i \omega_{0} \tau\right)+\sqrt{\varepsilon} v_{1, k}(\tau, \nu)+\varepsilon v_{2, k}(\tau, \nu)+\cdots, \\
r_{j, k}(\varepsilon, \nu) & =r_{j, k}^{0}(\nu)+\varepsilon r_{j, k}^{1}(\nu)+\cdots, \quad j=1,2 .
\end{aligned}
$$

Собственно говоря, в дальнейшем нам потребуется только явное выражение для матрицы $\Lambda_{k}(0, \nu)$. Для его нахождения поступим следующим образом. Подставим соотношение (2.6) в (2.5) и будем приравнивать коэффициенты при одинаковых степенях $\varepsilon$. В результате для фигурирующих в (2.8) вектор-функций $v_{m, k}, m \geqslant 1$, получаем рекуррентную последовательность линейных неоднородных уравнений вида

$$
\frac{d v_{m, k}}{d \tau}=A_{0} v_{m, k}+f_{m}(\tau)
$$

а неизвестные постоянные $r_{j, k}^{n}(\nu), n \geqslant 1$, определяем из условий их разрешимости в классе $2 \pi / \omega_{0}$-периодических функций. Несложный подсчет с использованием формул (2.2), (2.3) показывает, что система (2.9) при $m=1$ разрешима в требуемом классе автоматически, а разрешимости аналогичной системы при $m=2$ добиваемся за счет выбора элементов матрицы $\Lambda_{k}(0, \nu)$. На этом пути, в частности, приходим к равенству

$$
\Lambda_{k}(0, \nu)=C_{k}(\nu)
$$

где $C_{k}(\nu)$ - введенная выше матрица (1.24).

Из формул $(2.7),(2.10)$ и (1.24) следует, что всегда $\operatorname{tr} \Lambda_{k}(\varepsilon, \nu)<0$. Поэтому для нахождения требуемого критического значения параметра $\nu$ обратимся к случаю $k=1$ и рассмотрим равенство

$$
\operatorname{det} \Lambda_{1}(\nu, \varepsilon)=0 .
$$

Привлекая в очередной раз формулы (2.10), (1.24), убеждаемся, что в точке $\nu=\nu_{*}, \varepsilon=0$ для уравнения (2.11) выполняются условия теоремы о неявной 
функции по переменной $\nu$. Тем самым, из (2.11) однозначно определяется непрерывная по $\varepsilon$ функция $\nu=\nu(\varepsilon), \nu(0)=\nu_{*}$, раскладывающаяся в асимптотический ряд по целым степеням $\varepsilon$.

Проделанные предварительные построения позволяют перейти к строгой постановке бифуркационной проблемы для краевой задачи (1.1). Для того чтобы сделать это, учтем в ней равенство (1.19) и положим $\nu=\nu(\varepsilon)-\mu, 0<\mu \ll 1$, где, как и в (1.26), $\mu$ - новый малый параметр, отвечающий за отклонение $\nu$ от критического значения $\nu(\varepsilon)$. Однако в отличие от ситуации, рассмотренной в теореме 1.3, теперь параметры $\varepsilon$ и $\mu$ меняются независимо друг от друга. В результате получим краевую задачу

$$
\frac{\partial u}{\partial t}=\varepsilon D(\varepsilon, \mu) \frac{\partial^{2} u}{\partial x^{2}}+A(\varepsilon) u+F(u, \varepsilon),\left.\quad \frac{\partial u}{\partial x}\right|_{x=0}=\left.\frac{\partial u}{\partial x}\right|_{x=\pi}=0
$$

в которой $D(\varepsilon, \mu)=(\nu(\varepsilon)-\mu) D_{0}$.

Уместно отметить, что здесь нами реализован простейший способ введения дополнительного бифуркационного параметра $\mu$. Что же касается более общего подхода к выбору матрицы диффузии $D(\varepsilon, \mu)$, то он описывается в $\S 3$.

Справедливо следующее утверждение, являющееся основным результатом настоящей статьи.

ТЕОРема 2.1. Предположим, что в дополнение $\kappa$ перечисленным ранее ограничениям на матрицы $D_{0}, A(\varepsilon)$ и вектор-функцию $F(u, \varepsilon)$ собственные значения $\lambda_{k}, k=1, \ldots, N$, матрицы $A_{0}$ удовлетворяют условиям нерезонансности

$$
\lambda_{k} \neq \sum_{s=1}^{N} m_{s} \lambda_{s}, \quad k=1, \ldots, N,
$$

при любом иелочисленном векторе $\left(m_{1}, \ldots, m_{N}\right): \sum_{s=1}^{N}\left|m_{s}\right|=2$. Тогда найдутся такие $\varepsilon_{0}, \mu_{0}>0$, что при всех $0<\varepsilon \leqslant \varepsilon_{0}, 0<\mu \leqslant \mu_{0}$ краевая задача (2.12) имеет два экспоненциально орбитально устойчивых пространственно неоднородных периодических по t решения, располагающихся в некоторой асимптотически малой (порядка $\sqrt{\varepsilon \mu})$ окрестности неустойчивого однородного иикла (1.9).

2.2. Алгоритмическая часть доказательства. Проведем обоснование теоремы 2.1 сначала без предположения о нерезонансности (2.13), но при дополнительном условии

$$
F_{2}(u, u) \equiv 0,
$$

где $F_{2}$ - фигурирующая в (1.3) квадратичная нелинейность. Как будет ясно из дальнейшего анализа, это условие по существу эквивалентно требованиям (2.13).

Уместно отметить, что в случае (2.14) несколько упрощаются все возникающие при исследовании краевой задачи (1.1) асимптотические формулы. Например, обращаются в нуль функции $u_{2}(\tau), u_{2}(t, s, x)$ из $(1.9),(1.11)$, матрица $A_{0,1}(\tau)$ из $(2.2)$, а также фигурирующие в (2.8) вектор-функции $v_{1, k}(\tau, \nu)$. 
Алгоритмическая часть доказательства теоремы 2.1 заключается в построении асимптотики (пока формальной) пространственно неоднородных циклов краевой задачи (2.12), бифурцирующих из однородного цикла (1.9). Для того чтобы осуществить эту процедуру, необходимо выявить некоторые дополнительные свойства линейных систем

$$
\mathscr{L}_{k} h \equiv \sigma(\varepsilon) \frac{d h}{d \tau}-\left[A(\tau, \varepsilon)-\varepsilon k^{2} \nu(\varepsilon) D_{0}\right] h=0, \quad k=0,1, \ldots,
$$

получающихся из (2.5) при $\nu=\nu(\varepsilon)$. А именно, в первую очередь следует разобраться с вопросами существования у них при $k=0,1$ нетривиальных $2 \pi / \omega_{0}$-периодических решений и об обратимости дифференциальных операторов $\mathscr{L}_{0}, \mathscr{L}_{1}$ в подходящих пространствах периодических функций.

ЛЕмма 2.1. Пусть выполнены все перечисленные в предыдущем параграфе ограничения и дополнительное условие (2.14). Тогда, во-первых, уравнения $\mathscr{L}_{k} h=0, k=0,1$, и сопряженные уравнения $\mathscr{L}_{k}^{*} g=0, k=0,1$, допускают нетривиальные $2 \pi / \omega_{0}$-периодические решения $h_{0}(\tau, \varepsilon)=\varepsilon^{-1 / 2} d u_{*} / d \tau, h_{1}(\tau, \varepsilon)$ u $g_{0}(\tau, \varepsilon), g_{1}(\tau, \varepsilon)$ соответственно, нормированные условиями

$$
\left(h_{k}(\tau, \varepsilon), g_{k}(\tau, \varepsilon)\right) \equiv 1, \quad k=0,1 .
$$

Кроме того, за исключением простых единичных все осталъные мультипликаторь уравнений (2.15) при $k=0,1$ по модулю строго менъше единицы. Во-вторых, для фигурирующих в (2.16) периодических функций справедливы равномерные по т асимптотические представления

$$
\begin{aligned}
h_{0}(\tau, \varepsilon) & =i \omega_{0} \xi_{0}\left(a \exp \left(i \omega_{0} \tau\right)-\bar{a} \exp \left(-i \omega_{0} \tau\right)\right)+O(\varepsilon), \\
g_{0}(\tau, \varepsilon)= & \frac{1}{2 \omega_{0} \xi_{0}}\left(i\left(1+i y_{0}\right) b \exp \left(i \omega_{0} \tau\right)-i\left(1-i y_{0}\right) \bar{b} \exp \left(-i \omega_{0} \tau\right)\right)+O(\varepsilon), \\
h_{1}(\tau, \varepsilon)= & \xi_{0}\left(\left(\gamma_{1}+i \gamma_{2}\right) a \exp \left(i \omega_{0} \tau\right)+\left(\gamma_{1}-i \gamma_{2}\right) \bar{a} \exp \left(-i \omega_{0} \tau\right)\right)+O(\varepsilon), \\
g_{1}(\tau, \varepsilon)=- & \frac{1}{2\left(\gamma_{2} x_{0}-\gamma_{1}\right) \xi_{0}}\left(\left(1-i x_{0}\right) b \exp \left(i \omega_{0} \tau\right)\right. \\
& \left.+\left(1+i x_{0}\right) \bar{b} \exp \left(-i \omega_{0} \tau\right)\right)+O(\varepsilon) .
\end{aligned}
$$

В-третъих, каждое из линейных неоднородных уравнений

$$
\mathscr{L}_{k} h=f_{k}(\tau), \quad k=0,1,
$$

где $f_{k}(\tau)$ - произвольная непрерывная $2 \pi / \omega_{0}$-периодическая вектор-функиия, удовлетворяющая соответствующему условию разрешимости

$$
\int_{0}^{2 \pi / \omega_{0}}\left(f_{k}(\tau), g_{k}(\tau, \varepsilon)\right) d \tau=0,
$$

имеет единственное $2 \pi / \omega_{0}$-периодическое решение $h_{f_{k}}(\tau, \varepsilon)$, нормированное условием

$$
\int_{0}^{2 \pi / \omega_{0}}\left(h_{f_{k}}(\tau, \varepsilon), g_{k}(\tau, \varepsilon)\right) d \tau=0
$$


и допускающее оценку вида

$$
\max _{-\infty<\tau<\infty}\left\|h_{f_{k}}(\tau, \varepsilon)\right\| \leqslant \frac{M}{\varepsilon} \max _{-\infty<\tau<\infty}\left\|f_{k}(\tau)\right\|, \quad k=0,1 .
$$

Здесъ $\|*\|-$ евклидова норма, а постоянная $M>0$ является универсальной, т.е. не зависит ни от $\varepsilon$, ни от выбора неоднородности $f_{k}$.

ДокАзАтельство. Справедливость всех перечисленных в лемме утверждений так или иначе вытекает из уже проделанных ранее построений. Действительно, существование периодических решений $h_{k}$ и $g_{k}, k=0,1$, с требуемыми свойствами (2.16) очевидно, так как система (2.15) при $k=0$ представляет собой линеаризацию точечной модели (1.8) на устойчивом цикле (1.9), а значит, должна иметь простой единичный мультипликатор. Что же касается системы (2.15) при $k=1$, то наличие у нее простого единичного мультипликатора следует из способа выбора критического значения $\nu(\varepsilon)$ параметра $\nu$ (см. соответствующее место в п. 2.1).

Отметим, далее, что помимо простых единичных все остальные мультипликаторы систем (2.15) при $k=0,1$ по модулю строго меньше единицы. Точнее говоря, из описанного выше алгоритма исследования устойчивости заключаем, что у этих систем существуют так называемые некритические характеристические показатели (деленные на период логарифмы мультипликаторов), лежащие в комплексной полуплоскости вида $\{\lambda: \operatorname{Re} \lambda \leqslant-\delta\}$, где $\delta=$ const $>0$, и два критических показателя, являющихся собственными значениями матриц (см. (2.6)),

$$
\varepsilon \Lambda_{k}(\varepsilon, \nu(\varepsilon)), \quad k=0,1 .
$$

Остается добавить, что каждая из матриц (2.25) имеет вещественные собственные значения, одно из которых нулевое, а другое в силу равенств $(2.10),(1.24)$ является отрицательным.

Обратимся теперь к представлениям (2.17)-(2.20) и заметим, что поскольку в качестве $h_{0}(\tau, \varepsilon)$ мы берем $\varepsilon^{-1 / 2} d u_{*} / d \tau$, где $u_{*}(\tau, \varepsilon)-$ функция из $(1.9)$, то первое из них - следствие формул $(1.10)$ и тождества $u_{2}(\tau) \equiv 0$, имеющего место в силу (2.14). Далее, функция $h_{1}(\tau, \varepsilon)$ принадлежит, очевидно, двумерному критическому подпространству решений системы $\mathscr{L}_{1} h=0$, и в силу этого из (2.6) для нее вытекает соотношение

$$
h_{1}(\tau, \varepsilon)=V_{1}(\tau, \varepsilon, \nu(\varepsilon)) e_{1}(\varepsilon)
$$

где $e_{1}(\varepsilon)=\xi_{0}\left(\gamma_{1}+i \gamma_{2}, \gamma_{1}-i \gamma_{2}\right)^{T}+O(\varepsilon)$ - собственный вектор матрицы $(2.25)$ при $k=1$, отвечающий ее нулевому собственному значению. А отсюда с учетом свойств $(2.8),(2.10)$ и того факта, что $v_{1, k}(\tau, \nu) \equiv 0$, получаем требуемое равенство (2.19). Оставшиеся же асимптотические равенства (2.18) и (2.20) суть следствия аналогичных (2.26) представлений для периодических решений $g_{k}(\tau, \varepsilon), k=0,1$, сопряженных систем $\mathscr{L}_{k}^{*} g=0, k=0,1$. Анализ последних опустим, так как он проводится в точности по описанной в п. 2.1 схеме.

Для завершения обоснования леммы заметим, что при получении оценок (2.24) достаточно рассмотреть вместо (2.21) соответствующие уравнения 
на двумерных критических инвариантных подпространствах. После замен, приводящих матрицы $(2.25)$ к диагональной форме $\operatorname{diag}\left\{\varepsilon \lambda_{k}(\varepsilon), 0\right\}, \lambda_{k}(0)<0$, упомянутые уравнения приобретают вид

$$
\frac{d w_{1}}{d \tau}=\varepsilon \lambda_{k}(\varepsilon) w_{1}+f_{1, k}(\tau), \quad \frac{d w_{2}}{d \tau}=f_{2, k}(\tau), \quad k=0,1
$$

где $f_{1, k}(\tau), f_{2, k}(\tau)$ - произвольные скалярные непрерывные $2 \pi / \omega_{0}$-периодические функции, вторая из которых удовлетворяет аналогичному (2.22) условию разрешимости

$$
\int_{0}^{2 \pi / \omega_{0}} f_{2, k}(\tau) d \tau=0 .
$$

Анализ уравнений (2.27) не вызывает затруднений, поскольку их периодические решения $w_{1, f_{k}}, w_{2, f_{k}}$, нормированные аналогичными (2.23) равенствами, выписываются явно и для них наличие оценок вида (2.24) проверяется непосредственно. Лемма 2.1 доказана.

Обратимся, далее, к уравнениям (2.15) при $k \geqslant 2$ и обозначим через $U_{k}(\tau, s)$, $U_{k}(s, s)=I$, их фундаментальные матрицы. При выполнении условий леммы 2.1 справедливо следующее утверждение.

Лемма 2.2. Найдутся такие положительные постоянные $\varepsilon_{0}, \delta_{0}, M$, что при всех $0<\varepsilon \leqslant \varepsilon_{0}, k \geqslant 2$ и при любой непрерывной $2 \pi / \omega_{0}$-периодической вектор-функции $f(\tau)$ имеют место оценки

$$
\begin{gathered}
\left\|U_{k}(\tau, s)\right\| \leqslant M \exp \left[-\varepsilon \delta_{0} k^{2}(\tau-s)\right], \quad-\infty<s \leqslant \tau<\infty, \\
\max _{-\infty<\tau<\infty}\left\|h_{f, k}(\tau, \varepsilon)\right\| \leqslant \frac{M}{\varepsilon \delta_{0} k^{2}} \max _{-\infty<\tau<\infty}\|f(\tau)\|,
\end{gathered}
$$

где $h_{f, k}$ есть единственное периодическое решение неоднородного уравнения $\mathscr{L}_{k} h=f(\tau)$.

ДокАЗАТЕЛьство. Заметим сразу, что в обосновании нуждается только неравенство (2.28). Действительно, если оно уже установлено, то уравнение $\mathscr{L}_{k} h=f(\tau)$ имеет единственное периодическое решение

$$
h_{f, k}(\tau, \varepsilon)=\int_{-\infty}^{\tau} U_{k}(\tau, s) f(s) d s,
$$

для которого требуемая оценка (2.29) вытекает из (2.28) очевидным образом.

Для доказательства свойства (2.28) рассмотрим интегральное уравнение

$$
U_{k}(\tau, s)=K(\tau-s)+\frac{\varepsilon}{\sigma(\varepsilon)} \int_{s}^{\tau} K(\tau-\theta) A_{1}(\theta, \varepsilon) U_{k}(\theta, s) d \theta,
$$

где $K(\tau)=\exp \left[\left(A_{0}-\varepsilon k^{2} \nu(\varepsilon) D_{0}\right) \tau / \sigma(\varepsilon)\right], A_{1}(\tau, \varepsilon)$ - матрица из (2.2) (при выводе этого уравнения учтено вытекающее из (2.14) равенство $\left.A_{0,1}(\tau) \equiv 0\right)$. Отметим, далее, что из наложенных на матрицы $-D_{0}$ и $A_{0}-z D_{0}, z>0$, ограничений 
следует существование таких положительных констант $M_{1}, \delta_{1}$, что при всех $\tau \geqslant 0, z>0$ выполняется оценка

$$
\left\|\exp \left[\left(A_{0}-z D_{0}\right) \tau\right]\right\| \leqslant M_{1} \exp \left(-z \delta_{1} \tau\right) .
$$

Учитывая ее в (2.30), приходим к неравенству

$$
v_{k}(\tau, s, \varepsilon) \leqslant M_{1}+\frac{\varepsilon}{\sigma(\varepsilon)} M_{1} M_{2} \int_{s}^{\tau} v_{k}(\theta, s, \varepsilon) d \theta,
$$

где

$$
v_{k}(\tau, s, \varepsilon)=\left\|U_{k}(\tau, s)\right\| \exp \left(\varepsilon k^{2} \frac{\nu(\varepsilon)}{\sigma(\varepsilon)} \delta_{1}(\tau-s)\right), \quad M_{2}=\max _{\tau, \varepsilon}\left\|A_{1}(\tau, \varepsilon)\right\|,
$$

из которого в свою очередь в силу леммы Гронуолла-Беллмана имеем

$$
\left\|U_{k}(\tau, s)\right\| \leqslant M_{1} \exp \left\{\left[-\varepsilon k^{2} \frac{\nu(\varepsilon)}{\sigma(\varepsilon)} \delta_{1}+\frac{\varepsilon}{\sigma(\varepsilon)} M_{1} M_{2}\right](\tau-s)\right\}, \quad \tau \geqslant s .
$$

Рассмотрим сначала все номера $k: k \geqslant k_{0}$, где $k_{0}$ выбрано из условия $k_{0}^{2} \nu_{*} \delta_{1}>M_{1} M_{2}$. Тогда, очевидно, полученная выше оценка (2.31) эквивалентна требуемому неравенству (2.28). Если же $2 \leqslant k \leqslant k_{0}$, то проблема сводится к анализу уравнений на двумерных критических инвариантных подпространствах систем (2.15). В комплексных переменных $\widetilde{w}=(w, \bar{w})^{T}$ эти уравнения принимают вид

$$
\frac{d \widetilde{w}}{d \tau}=\varepsilon \Lambda_{k}(\varepsilon, \nu(\varepsilon)) \widetilde{w}, \quad k=2, \ldots, k_{0},
$$

где $\Lambda_{k}$ - двумерные матрицы из (2.6). Остается заметить, что для каждого из уравнений (2.32) аналог неравенства (2.28) имеет место в силу гурвицевости матриц $\Lambda_{k}\left(0, \nu_{*}\right), k \geqslant 2$. Лемма 2.2 доказана.

Проделанный линейный анализ показывает, что при $\mu=0$ в задаче об устойчивости однородного цикла (1.9) системы (2.12) реализуется критический случай двукратного единичного мультипликатора без жордановой клетки, так как соответствующая линейная система (2.1) при $\nu=\nu(\varepsilon)$ допускает два независимых периодических решения

$$
h=h_{0}(\tau, \varepsilon), \quad h=h_{1}(\tau, \varepsilon) \cos x,
$$

где $h_{0}, h_{1}$ - функции $(2.17),(2.19)$. Тем самым, обретает смысл проблема нахождения пространственно неоднородных периодических решений краевой задачи (2.12), бифурцирующих при $\mu>0$ из однородного цикла.

Для построения асимптотики интересующих нас периодических решений выполним сначала в (2.12) замены

$$
\tau=\sigma(\varepsilon)(1+\varepsilon \delta(\varepsilon, \mu)) t, \quad u=u_{*}(\tau, \varepsilon)+\sqrt{\varepsilon} v,
$$

где $\delta(\varepsilon, \mu), \delta(\varepsilon, 0) \equiv 0,-$ некоторая подлежащая определению поправка к частоте, а $u_{*}(\tau, \varepsilon)$ - функция из (1.9). В результате для $v$ получаем краевую задачу 
Вида

$$
\begin{gathered}
\varepsilon \sigma(\varepsilon) \delta(\varepsilon, \mu) h_{0}(\tau, \varepsilon)+\sigma(\varepsilon)(1+\varepsilon \delta(\varepsilon, \mu)) \frac{\partial v}{\partial \tau} \\
=\varepsilon D(\varepsilon, \mu) \frac{\partial^{2} v}{\partial x^{2}}+A(\tau, \varepsilon) v+\varepsilon \Phi(\tau, v, \varepsilon), \\
\left.\frac{\partial v}{\partial x}\right|_{x=0}=\left.\frac{\partial v}{\partial x}\right|_{x=\pi}=0
\end{gathered}
$$

с $2 \pi / \omega_{0}$-периодическими по $\tau$ коэффициентами. Здесь $A(\tau, \varepsilon)$ - матрица $(2.2)$, а нелинейность $\Phi$ задается равенством

$$
\Phi(\tau, v, \varepsilon)=\left.\frac{1}{\varepsilon^{3 / 2}}\left(F(u+\sqrt{\varepsilon} v, \varepsilon)-F(u, \varepsilon)-\sqrt{\varepsilon} F_{u}^{\prime}(u, \varepsilon) v\right)\right|_{u=u_{*}(\tau, \varepsilon)},
$$

где $F(u, \varepsilon)$ - вектор-функция из (1.1).

Остановимся чуть более подробно на свойствах функции (2.36). Из равенств $(1.3),(1.9),(1.10)$ и (2.14) следует, что, во-первых, при $\varepsilon \rightarrow 0$ для нее справедлива асимптотическая формула

$$
\Phi(\tau, v, \varepsilon)=3 F_{3}\left(u_{1}(\tau), v, v\right)+F_{3}(v, v, v)+O(\sqrt{\varepsilon})
$$

сохраняющаяся при дифференцировании по $v$ любое фиксированное число раз и равномерная по $\tau \in \mathbb{R}, v \in \Omega$, где $\Omega \subset \mathbb{R}^{N}$ - произвольный компакт; во-вторых, ее тейлоровское разложение в точке $v=0$ имеет вид

$$
\Phi(\tau, v, \varepsilon)=\Phi_{2}(\tau, v, v, \varepsilon)+\Phi_{3}(\tau, v, v, v, \varepsilon)+\cdots,
$$

где функции $\Phi_{2}, \Phi_{3}$, являющиеся по $v$ симметричными квадратичной и кубической формами соответственно, допускают в свою очередь аналогичные (2.37) асимптотические представления

$$
\Phi_{2}=3 F_{3}\left(u_{1}(\tau), v, v\right)+O\left(\sqrt{\varepsilon}\|v\|^{2}\right), \quad \Phi_{3}=F_{3}(v, v, v)+O\left(\sqrt{\varepsilon}\|v\|^{3}\right),
$$

равномерные по $\tau \in \mathbb{R}, v \in \Omega$.

Возвращаясь к нашей основной проблеме и обыгрывая существование у системы $(2.1)$ при $\nu=\nu(\varepsilon)$ второго из периодических решений $(2.33)$, поправку к частоте $\delta(\varepsilon, \mu)$ из $(2.34)$ и возможные пространственно неоднородные периодические решения краевой задачи (2.35) будем искать в виде рядов по целым степеням $\mu$ и $\sqrt{\mu}$ соответственно:

$$
\begin{aligned}
\delta(\varepsilon, \mu) & =\delta_{1}(\varepsilon) \mu+\delta_{2}(\varepsilon) \mu^{2}+\cdots \\
v & =\sqrt{\mu} v_{0}(\tau, x, \varepsilon)+\mu v_{1}(\tau, x, \varepsilon)+\mu^{3 / 2} v_{2}(\tau, x, \varepsilon)+\cdots \\
v_{0} & =\eta_{0} h_{1}(\tau, \varepsilon) \cos x
\end{aligned}
$$

где $\eta_{0}=\eta_{0}(\varepsilon)$ - произвольная (подлежащая определению) вещественная амплитуда, все функции $v_{j}, j \geqslant 1$, периодичны по $\tau$ с периодом $2 \pi / \omega_{0}$. 
Дальнейший анализ во многом аналогичен изложенному в предыдущем параграфе алгоритму построения асимптотики автомодельных циклов квазинормальной формы. Как и в п. 1.3, подставим ряды $(2.40),(2.41)$ вместе с разложением (2.38) в систему (2.35) и будем последовательно приравнивать коэффициенты при одинаковых степенях $\mu$. В результате на первом шаге алгоритма для нахождения $\delta_{1}(\varepsilon)$ и $v_{1}(\tau, x, \varepsilon)$ приходим к краевой задаче

$$
\Pi(\varepsilon) v_{1}=\varepsilon f_{1}(\tau, x, \varepsilon),\left.\quad \frac{\partial v_{1}}{\partial x}\right|_{x=0}=\left.\frac{\partial v_{1}}{\partial x}\right|_{x=\pi}=0,
$$

где $\Pi(\varepsilon)=\sigma(\varepsilon) \partial / \partial \tau-\varepsilon \nu(\varepsilon) D_{0} \partial^{2} / \partial x^{2}-A(\tau, \varepsilon)$, а неоднородность $f_{1}$ задается равенством

$$
f_{1}=\eta_{0}^{2} \Phi_{2}\left(\tau, h_{1}(\tau, \varepsilon), h_{1}(\tau, \varepsilon), \varepsilon\right) \cos ^{2} x-\sigma(\varepsilon) \delta_{1}(\varepsilon) h_{0}(\tau, \varepsilon) .
$$

Обратимся сначала к условиям разрешимости задачи $(2.42)$ в классе $2 \pi / \omega_{0}$ периодических функций, имеющим вид

$$
\begin{array}{r}
\int_{0}^{2 \pi / \omega_{0}} \int_{0}^{\pi}\left(f_{1}, g_{0}(\tau, \varepsilon)\right) d x d \tau=0 \\
\int_{0}^{2 \pi / \omega_{0}} \int_{0}^{\pi}\left(f_{1}, g_{1}(\tau, \varepsilon)\right) \cos x d x d \tau=0
\end{array}
$$

где $g_{0}, g_{1}$ - функции $(2.18),(2.20)$. В силу (2.43) второе из них выполняется автоматически, а из первого находим неизвестную поправку к частоте $\delta_{1}(\varepsilon)$ :

$$
\begin{gathered}
\delta_{1}(\varepsilon)=\eta_{0}^{2} \delta_{1,0}(\varepsilon), \\
\delta_{1,0}(\varepsilon)=\frac{\omega_{0}}{4 \pi \sigma(\varepsilon)} \int_{0}^{2 \pi / \omega_{0}}\left(\Phi_{2}\left(\tau, h_{1}(\tau, \varepsilon), h_{1}(\tau, \varepsilon), \varepsilon\right), g_{0}(\tau, \varepsilon)\right) d \tau .
\end{gathered}
$$

Учитывая, далее, в (2.45) асимптотические представления (2.18), (2.19), (2.39), приходим к выводу, что

$$
\delta_{1,0}(\varepsilon)=-\frac{\phi_{0}}{\omega_{0}}\left(1+y_{0}^{2}\right) \gamma_{1} \gamma_{2}+O\left(\varepsilon^{1 / 2}\right) .
$$

После определения $\delta_{1}(\varepsilon)$ посредством равенств (2.45) краевая задача $(2.42)$ становится разрешимой в требуемом классе функций, а ее решение имеет вид

$$
v_{1}=\eta_{0}^{2}\left(v_{1,0}(\tau, \varepsilon)+v_{1,2}(\tau, \varepsilon) \cos 2 x\right),
$$

где вектор-функции

$$
v_{1,0}(\tau, \varepsilon), \quad \int_{0}^{2 \pi / \omega_{0}}\left(v_{1,0}(\tau, \varepsilon), g_{0}(\tau, \varepsilon)\right) d \tau=0, \quad v_{1,2}(\tau, \varepsilon)
$$

- периодические решения линейных систем

$$
\begin{aligned}
& \mathscr{L}_{0} v_{1,0}=\frac{\varepsilon}{2} \Phi_{2}\left(\tau, h_{1}(\tau, \varepsilon), h_{1}(\tau, \varepsilon), \varepsilon\right)-\varepsilon \sigma(\varepsilon) \delta_{1,0}(\varepsilon) h_{0}(\tau, \varepsilon), \\
& \mathscr{L}_{2} v_{1,2}=\frac{\varepsilon}{2} \Phi_{2}\left(\tau, h_{1}(\tau, \varepsilon), h_{1}(\tau, \varepsilon), \varepsilon\right) .
\end{aligned}
$$


Уместно напомнить, что в силу лемм 2.1, 2.2 интересующие нас решения систем (2.48) находятся однозначно, а из оценок (2.24), (2.29) вытекает их равномерная по $\tau \in \mathbb{R}$ ограниченность при $\varepsilon \rightarrow 0$. Однако для наших целей требуется знать асимптотическое поведение функций $v_{1,0}(\tau, \varepsilon), v_{1,2}(\tau, \varepsilon)$ более подробно. Необходимый объем информации об этих функциях содержится в следующем утверждении.

ЛЕмма 2.3. Пусть выполнены все перечисленные в лемме 2.1 ограничения. Тогда при $\varepsilon \rightarrow 0$ равномерно по $\tau \in \mathbb{R}$ справедливь асимптотические равенства

$$
v_{1,0}(\tau, \varepsilon)=v_{1,0}^{0}(\tau)+O(\sqrt{\varepsilon}), \quad v_{1,2}(\tau, \varepsilon)=v_{1,2}^{0}(\tau)+O(\sqrt{\varepsilon}),
$$

в которых

$$
\begin{aligned}
& v_{1,0}^{0}(\tau)=\xi_{0} R_{0}\left[\left(1+i y_{0}\right) a \exp \left(i \omega_{0} \tau\right)+\left(1-i y_{0}\right) \bar{a} \exp \left(-i \omega_{0} \tau\right)\right] \\
& v_{1,2}^{0}(\tau)=\xi_{0}\left[\left(R_{1}+i R_{2}\right) a \exp \left(i \omega_{0} \tau\right)+\left(R_{1}-i R_{2}\right) \bar{a} \exp \left(-i \omega_{0} \tau\right)\right]
\end{aligned}
$$

a $R_{j}, j=0,1,2,-$ постоянные из (1.38).

ДокАЗАТЕЛЬство. Обратимся сначала к первому уравнению из (2.48) и положим в нем

$$
v_{1,0}(\tau, \varepsilon)=v_{1,0}^{0}(\tau)+\varepsilon v_{1,0}^{1}(\tau)+\cdots, \quad \delta_{1,0}(\varepsilon)=\delta_{1,0}^{0}+\sqrt{\varepsilon} \delta_{1,0}^{1}+\cdots,
$$

где в силу (2.46) поправка $\delta_{1,0}^{0}$ задается равенством

$$
\delta_{1,0}^{0}=-\frac{\phi_{0}}{\omega_{0}}\left(1+y_{0}^{2}\right) \gamma_{1} \gamma_{2}
$$

функция $v_{1,0}^{0}(\tau)$ имеет вид

$$
v_{1,0}^{0}(\tau)=\varkappa a \exp \left(i \omega_{0} \tau\right)+\bar{\varkappa} \bar{a} \exp \left(-i \omega_{0} \tau\right)
$$

а $\varkappa$ - пока произвольная комплексная постоянная. Приравнивая затем коэффициенты $\varepsilon$ и учитывая асимптотическое представление (2.2), в котором, напомним, $A_{0,1}(\tau) \equiv 0$, для нахождения $v_{1,0}^{1}$ получаем линейную неоднородную систему вида

$$
\frac{d v_{1,0}^{1}}{d \tau}=A_{0} v_{1,0}^{1}+f_{1,0}^{1}(\tau)
$$

где

$$
f_{1,0}^{1}=\frac{1}{2} \Phi_{2}\left(\tau, h_{1}(\tau, 0), h_{1}(\tau, 0), 0\right)-\delta_{1,0}^{1} h_{0}(\tau, 0)-\alpha_{0} \frac{d v_{1,0}^{0}}{d \tau}+A_{1}(\tau, 0) v_{1,0}^{0}(\tau),
$$

$\alpha_{0}$ - поправка к частоте однородного цикла (см. (1.10)).

Дальнейший способ действий стандартен: поскольку нас интересует $2 \pi / \omega_{0}$ периодическое решение системы (2.54), то мы приравниваем к нулю скалярное произведение коэффициента неоднородности (2.55) при $\exp \left(i \omega_{0} \tau\right)$ на вектор $b$. В результате с учетом формул $(1.10),(2.17),(2.19),(2.39),(2.52)$ и явного вида 
матрицы $A_{1}(\tau, 0)$ (см. $\left.(2.3)\right)$ для отыскания фигурирующей в $(2.53)$ постоянной $\varkappa$ получается уравнение

$$
\varkappa+\bar{\varkappa}=\frac{\xi_{0}}{2}\left(2 y_{0} \gamma_{1} \gamma_{2}-3 \gamma_{1}^{2}-\gamma_{2}^{2}\right) .
$$

Обратим внимание на то, что возможные решения уравнения (2.56) определяются с точностью до слагаемого $i c, c \in \mathbb{R}$. Но так как функция $v_{1,0}(\tau, \varepsilon)$ удовлетворяет условию нормировки вида (2.23) (при $k=0)$, то в силу $(2.18),(2.51)$ мы должны положить

$$
\varkappa=\xi_{0}\left(1+i y_{0}\right) R_{0} .
$$

Обоснование изложенных формальных построений проводится по следующей схеме. Подставим в первое уравнение из (2.48) соотношения

$$
v_{1,0}=v_{1,0}^{0}(\tau)+\varepsilon v_{1,0}^{1}(\tau)+h, \quad \delta_{1,0}(\varepsilon)=\delta_{1,0}^{0}+\sqrt{\varepsilon} \delta_{*}(\varepsilon),
$$

где нулевое приближение $v_{1,0}^{0}(\tau)$ определено формулами $(2.53),(2.57), \delta_{1,0}^{0}$ - величина (2.52), а $v_{1,0}^{1}$ - некоторое фиксированное $2 \pi / \omega_{0}$-периодическое решение системы (2.54). В итоге для нахождения $h$ получаем уравнение вида

$$
\mathscr{L}_{0} h=\varepsilon^{3 / 2}\left(\tilde{f}(\tau, \varepsilon)-\sigma(\varepsilon) \delta_{*}(\varepsilon) h_{0}(\tau, \varepsilon)\right)
$$

где $2 \pi / \omega_{0}$-периодическая неоднородность $\tilde{f}$ остается ограниченной при $\varepsilon \rightarrow 0$. Полагая, далее, в (2.59)

$$
\delta_{*}(\varepsilon)=\frac{\omega_{0}}{2 \pi \sigma(\varepsilon)} \int_{0}^{2 \pi / \omega_{0}}\left(\widetilde{f}(\tau, \varepsilon), g_{0}(\tau, \varepsilon)\right) d \tau,
$$

сначала находим $\widetilde{h}(\tau, \varepsilon)=\varepsilon^{3 / 2} \mathscr{L}_{0}^{-1}\left(\tilde{f}-\sigma(\varepsilon) \delta_{*}(\varepsilon) h_{0}\right)=O(\sqrt{\varepsilon})$ (см. (2.24) при $k=0)$, а затем и фигурирующую в (2.58) добавку

$$
h=\widetilde{h}(\tau, \varepsilon)+c(\varepsilon) h_{0}(\tau, \varepsilon), \quad c(\varepsilon)=-\frac{\omega_{0}}{2 \pi} \int_{0}^{2 \pi / \omega_{0}}\left(v_{1,0}^{0}+\varepsilon v_{1,0}^{1}, g_{0}\right) d \tau=O(\varepsilon)
$$

имеющую равномерно по $\tau \in \mathbb{R}$ порядок малости $\sqrt{\varepsilon}$. Тем самым, установлено первое из асимптотических равенств (2.49). Второе из них обосновывается аналогично. Лемма 2.3 доказана.

На втором шаге алгоритма нахождения коэффициентов рядов (2.40), (2.41) для $v_{2}(\tau, x, \varepsilon)$ получаем аналогичную (2.42) краевую задачу

$$
\Pi(\varepsilon) v_{2}=\varepsilon f_{2}(\tau, x, \varepsilon),\left.\quad \frac{\partial v_{2}}{\partial x}\right|_{x=0}=\left.\frac{\partial v_{2}}{\partial x}\right|_{x=\pi}=0,
$$

где

$$
f_{2}=2 \Phi_{2}\left(\tau, v_{0}, v_{1}, \varepsilon\right)+\Phi_{3}\left(\tau, v_{0}, v_{0}, v_{0}, \varepsilon\right)-\sigma(\varepsilon) \delta_{1}(\varepsilon) \frac{\partial v_{0}}{\partial \tau}-D_{0} \frac{\partial^{2} v_{0}}{\partial x^{2}}
$$

Подставляя, далее, в (2.61) явные выражения для функций $v_{0}, v_{1}$ (см. (2.41), $(2.47))$, приходим к выводу, что неоднородность $f_{2}$ автоматически удовлетворяет первому из условий разрешимости вида (2.44). Второе же из этих условий, 
как и в п. 1.3, будем рассматривать в качестве уравнения для отыскания неизвестной амплитуды $\eta_{0}$.

После некоторых преобразований упомянутое уравнение принимает аналогичный (1.39) вид

$$
\beta_{1}(\varepsilon) \eta_{0}+\beta_{2}(\varepsilon) \eta_{0}^{3}=0
$$

где

$$
\begin{aligned}
\beta_{1}(\varepsilon)= & \frac{\omega_{0}}{2 \pi} \int_{0}^{2 \pi / \omega_{0}}\left(D_{0} h_{1}(\tau, \varepsilon), g_{1}(\tau, \varepsilon)\right) d \tau \\
\beta_{2}(\varepsilon)= & \frac{\omega_{0}}{2 \pi} \int_{0}^{2 \pi / \omega_{0}}\left[-\sigma(\varepsilon) \delta_{1,0}(\varepsilon) \frac{\partial h_{1}}{\partial \tau}+2 \Phi_{2}\left(\tau, h_{1}, v_{1,0}, \varepsilon\right)\right. \\
& \left.+\Phi_{2}\left(\tau, h_{1}, v_{1,2}, \varepsilon\right)+\frac{3}{4} \Phi_{3}\left(\tau, h_{1}, h_{1}, h_{1}, \varepsilon\right)\right] g_{1}(\tau, \varepsilon) d \tau
\end{aligned}
$$

а функции $\delta_{1,0}(\varepsilon), v_{1,0}(\tau, \varepsilon), v_{1,2}(\tau, \varepsilon)$ те же самые, что и в $(2.45),(2.47)$. Более того, опираясь на асимптотические представления (2.19), (2.20), (2.46), (2.49), (2.50), убеждаемся, что при $\varepsilon \rightarrow 0$ коэффициенты (2.63) стремятся к конечным пределам

$$
\beta_{1}(0)=\frac{\left(x_{0} y_{0}-1\right)\left(1+x_{0}^{2}\right)}{\gamma_{2} x_{0}-\gamma_{1}}>0, \quad \beta_{2}(0)=\frac{\phi_{0} Q\left(x_{0}, y_{0}\right)}{\gamma_{2} x_{0}-\gamma_{1}}<0,
$$

где $Q\left(x_{0}, y_{0}\right)$ - функция (1.40). А это, в свою очередь, означает, что уравнение разветвления (2.62) имеет нетривиальное решение

$$
\eta_{0}(\varepsilon)=\sqrt{-\frac{\beta_{1}(\varepsilon)}{\beta_{2}(\varepsilon)}}
$$

После определения амплитуды $\eta_{0}$ посредством равенства (2.65) неоднородность (2.61) принимает вид

$$
f_{2}=f_{2,1}(\tau, \varepsilon) \cos x+f_{2,3}(\tau, \varepsilon) \cos 3 x, \quad \int_{0}^{2 \pi / \omega_{0}}\left(f_{2,1}(\tau, \varepsilon), g_{1}(\tau, \varepsilon)\right) d \tau=0
$$

В таком же виде ищем и решение $v_{2}$ краевой задачи (2.60). В результате приходим к аналогичному (2.66) равенству

$$
v_{2}=v_{2,1}(\tau, \varepsilon) \cos x+v_{2,3}(\tau, \varepsilon) \cos 3 x, \quad \int_{0}^{2 \pi / \omega_{0}}\left(v_{2,1}(\tau, \varepsilon), g_{1}(\tau, \varepsilon)\right) d \tau=0
$$

где $v_{2,1}, v_{2,3}$ - периодические решения соответствующих линейных систем

$$
\mathscr{L}_{1} v_{2,1}=\varepsilon f_{2,1}, \quad \mathscr{L}_{3} v_{2,3}=\varepsilon f_{2,3} .
$$

Напомним, что существование и единственность таких решений гарантируют леммы 2.1, 2.2. Более того, можно показать, что здесь справедлив аналог леммы 2.3 , т.е. эти решения стремятся при $\varepsilon \rightarrow 0$ к некоторым предельным 
функциям. Однако для дальнейшего важен только факт равномерной по $\varepsilon$ ограниченности $v_{2,1}(\tau, \varepsilon), v_{2,3}(\tau, \varepsilon)$, вытекающий из оценок $(2.24),(2.29)$.

Завершая описание алгоритмической части, отметим, что построение рядов (2.40), (2.41) может быть продолжено неограниченно, причем все функции $v_{j}, j \geqslant 1$, будут иметь конечные пределы при $\varepsilon \rightarrow 0$. Например, для нахождения $v_{3}$ и $v_{4}$ необходимо в формуле (2.67) учесть дополнительное слагаемое вида $\eta_{1} h_{1}(\tau, \varepsilon) \cos x$, где $\eta_{1}$ - еще одна произвольная вещественная амплитуда. Что же касается неизвестных постоянных $\delta_{2}$ и $\eta_{1}$, то они определяются из условий разрешимости линейных неоднородных краевых задач для $v_{3}$ и $v_{4}$ соответственно. При этом, что характерно, для $\eta_{1}$ и всех других аналогичных амплитуд, возникающих на шагах с четными номерами, вместо (2.62) получаются уже линейные неоднородные уравнения вида $2 \beta_{1}(\varepsilon) \eta_{1}=\varphi_{1}$.

2.3. Существование пространственно неоднородных циклов. В этом пункте проведем обоснование изложенного выше формализма, т.е. покажем существование у краевой задачи (2.12) при дополнительном условии (2.14) двух пространственно неоднородных циклов с асимптотикой вида (2.34), (2.40), (2.41). Но сначала выявим некоторые необходимые для дальнейшего свойства дифференциального оператора П $(\varepsilon)$ из $(2.42)$, дополненного нулевыми граничными условиями Неймана.

Обозначим через $E_{0}$ банахово пространство $2 \pi / \omega_{0}$-периодических по $\tau$ вектор-функций $u(\tau, x)$, принимающих при каждом $\tau$ значения из соболевского пространства $W_{2}^{1}\left([0, \pi] ; \mathbb{R}^{N}\right)$ и непрерывных по $\tau$ в метрике этого пространства. Норму в $E_{0}$ определим следующим образом: пусть

$$
u(\tau, x)=\sum_{m=0}^{\infty} u_{m}(\tau) \widetilde{c}_{m} \cos m x, \quad r_{m}=\max _{\tau}\left\|u_{m}(\tau)\right\|_{\mathbb{R}^{N}}
$$

где $\widetilde{c}_{m}=\sqrt{2 /\left(\pi\left(m^{2}+1\right)\right)}, m \geqslant 1, \widetilde{c}_{0}=\sqrt{1 / \pi}$. Тогда по определению

$$
\|u\|_{E_{0}}=\left(\sum_{m=0}^{\infty} r_{m}^{2}\right)^{1 / 2} .
$$

Далее нам потребуется еще пространство $E_{1} \subset E_{0}$, состоящее из таких функций $u(\tau, x)$, что $\partial u / \partial \tau, \partial u / \partial x, \partial^{2} u / \partial x^{2} \in E_{0}$ и $\partial u /\left.\partial x\right|_{x=0, \pi}=0$. Норму в $E_{1}$ определим аналогичной (2.69) формулой

$$
\|u\|_{E_{1}}=\left(\|u\|_{E_{0}}^{2}+\left\|\frac{\partial^{2} u}{\partial x^{2}}\right\|_{E_{0}}^{2}+\left\|\frac{\partial u}{\partial \tau}\right\|_{E_{0}}^{2}\right)^{1 / 2} .
$$

И наконец, через $\widetilde{E}_{j} \subset E_{j}, j=0,1$, обозначим подпространства функций, удовлетворяющих дополнительным равенствам

$$
\begin{aligned}
\int_{0}^{2 \pi / \omega_{0}} & \int_{0}^{\pi}\left(u(\tau, x), g_{0}(\tau, \varepsilon)\right) d x d \tau \\
= & \int_{0}^{2 \pi / \omega_{0}} \int_{0}^{\pi}\left(u(\tau, x), g_{1}(\tau, \varepsilon)\right) \cos x d x d \tau=0 .
\end{aligned}
$$


Возвращаясь к оператору П $(\varepsilon)$, рассмотрим неоднородное уравнение

$$
\Pi(\varepsilon) u=f(\tau, x)
$$

с произвольной правой частью $f \in \widetilde{E}_{0}$. Подставим затем в $(2.72)$ разложение (2.68) вместе с аналогичным рядом

$$
f(\tau, x)=\sum_{m=0}^{\infty} f_{m}(\tau) \widetilde{c}_{m} \cos m x
$$

для $f$. В результате приходим к счетной системе обыкновенных уравнений

$$
\mathscr{L}_{m} u_{m}=f_{m}(\tau), \quad m \geqslant 0,
$$

удовлетворяющей условиям лемм 2.1, 2.2. В частности, в силу $(2.71)$ для $f_{0}(\tau)$ и $f_{1}(\tau)$ выполняются требуемые условия разрешимости вида (2.22). Тем самым, каждое из уравнений (2.73) имеет единственное периодическое решение, для которого справедлива оценка (2.24) или (2.29). А отсюда и из способа введения норм в $E_{0}, E_{1}$ (см. $\left.(2.69),(2.70)\right)$ заключаем, что уравнение $(2.72)$ имеет единственное $2 \pi / \omega_{0}$-периодическое решение $u_{f}(\tau, x, \varepsilon) \in \widetilde{E}_{1}$, удовлетворяющее неравенству

$$
\left\|u_{f}\right\|_{E_{1}} \leqslant \frac{M}{\varepsilon}\|f\|_{E_{0}}
$$

с некоторой не зависящей от $\varepsilon, f$ постоянной $M>0$.

Перейдем теперь непосредственно к обоснованию асимптотических представлений $(2.40),(2.41)$. В связи с этим обозначим через $v_{0, *}(\tau, x, \varepsilon), v_{1, *}(\tau, x, \varepsilon)$ коэффициенты $v_{0}, v_{1}$ разложения $(2.41)$, вычисленные при $\eta_{0}=\eta_{0}(\varepsilon)$, где $\eta_{0}(\varepsilon)$ - амплитуда (2.65). Справедливо следующее утверждение.

ЛЕмма 2.4. При выполнении условий леммы 2.1 найдутся такие достаточно малье $\varepsilon_{0}, \mu_{0}>0$, что при всех $0<\varepsilon \leqslant \varepsilon_{0}, 0<\mu \leqslant \mu_{0}$ существуют две пары функиий

$$
\begin{aligned}
\left(\delta(\varepsilon, \mu), v_{ \pm}(\tau, x, \varepsilon, \mu)\right): \quad v_{-}(\tau, x, \varepsilon, \mu) & =v_{+}(\tau, \pi-x, \varepsilon, \mu), \\
v_{+}\left(\tau+\frac{2 \pi}{\omega_{0}}, x, \varepsilon, \mu\right) & =v_{+}(\tau, x, \varepsilon, \mu)
\end{aligned}
$$

обращающих краевую задачу (2.35) в тождество. При этом для $\delta(\varepsilon, \mu) u$ $v_{+}(\tau, x, \varepsilon, \mu)$ справедливы асимптотические представления вида

$$
\begin{gathered}
\delta(\varepsilon, \mu)=\eta_{0}^{2}(\varepsilon) \delta_{1,0}(\varepsilon) \mu+\delta_{*}(\varepsilon, \mu) \mu^{2}, \\
v_{+}=\sqrt{\mu} v_{0, *}(\tau, x, \varepsilon)+\mu v_{1, *}(\tau, x, \varepsilon)+\mu^{3 / 2} h_{*}(\tau, x, \varepsilon, \mu), \quad h_{*} \in E_{1},
\end{gathered}
$$

где $\left|\delta_{*}(\varepsilon, \mu)\right| \leqslant M_{1},\left\|h_{*}\right\|_{E_{1}} \leqslant M_{2}$, а постоянные $M_{1}, M_{2}>0$ не зависят от $\varepsilon, \mu$.

ДокАЗАТЕЛЬСтво. Подставим в (2.35) выражения

$$
\delta(\varepsilon, \mu)=\eta_{0}^{2} \delta_{1,0}(\varepsilon) \mu+\widetilde{\delta} \mu^{3 / 2}, \quad v=\sqrt{\mu} v_{0}+\mu v_{1}+\mu^{3 / 2} h, \quad h \in \widetilde{E}_{1},
$$


где $\widetilde{\delta}$ - некоторый произвольный параметр, функции $v_{0}, v_{1}$ определены равенствами из (2.41), (2.47), а фигурирующая в них амплитуда $\eta_{0}$ также пока произвольна (считаем, что параметры $\widetilde{\delta}, \eta_{0}$ меняются на некоторых фиксированных компактных множествах). В результате для неизвестной вектор-функции $h \in \widetilde{E}_{1}$ приходим к уравнению вида

$$
\Pi(\varepsilon) h=\varepsilon f\left(\tau, x, \varepsilon, \eta_{0}\right)-\varepsilon \sigma(\varepsilon) \widetilde{\delta} h_{0}(\tau, \varepsilon)+\varepsilon \sqrt{\mu} \Psi\left(\tau, x, \varepsilon, \mu, \eta_{0}, \widetilde{\delta}, h, \frac{\partial^{2} h}{\partial x^{2}}\right) .
$$

Здесь вектор-функция $f$ задается формулой (2.61), а явный вид функции $\Psi\left(\tau, x, \varepsilon, \mu, \eta_{0}, \widetilde{\delta}, u, v\right)$ для нас не представляет интереса. Важно лишь то, что, во-первых, она непрерывна по совокупности переменных (вплоть до значений $\mu=0, \varepsilon=0)$ вместе с любым наперед заданным количеством своих производных по $\left(\eta_{0}, \widetilde{\delta}, u, v\right) \in \mathbb{R} \times \mathbb{R} \times \mathbb{R}^{N} \times \mathbb{R}^{N} ;$ во-вторых, при произвольных допустимых значениях $\varepsilon, \mu, \eta_{0}, \widetilde{\delta}$ оператор суперпозиции $h \rightarrow \Psi\left(\tau, x, \varepsilon, \mu, \eta_{0}, \widetilde{\delta}, h, \partial^{2} h / \partial x^{2}\right)$ действует из $E_{1}$ в $E_{0}$ и является достаточно гладким по Фреше.

Анализ уравнения (2.78) проводится по следующей стандартной схеме. Сначала подправим его правую часть (которую ниже для краткости обозначаем через $\varepsilon G)$ так, чтобы при подстановке в нее $h \in \widetilde{E}_{1}$ получался элемент пространства $\widetilde{E}_{0}$, а затем обратим оператор П( $\varepsilon$ ) (что возможно в силу $(2.74)$ ). В результате приходим к интегральному уравнению в пространстве $\widetilde{E}_{1}$ вида

$$
h=\varepsilon \Pi^{-1}(\varepsilon)\left(G-l_{1}(G) h_{0}(\tau, \varepsilon)-l_{2}(G) h_{1}(\tau, \varepsilon) \cos x\right),
$$

где

$$
\begin{aligned}
& l_{1}(G)=\frac{\omega_{0}}{2 \pi^{2}} \int_{0}^{2 \pi / \omega_{0}} \int_{0}^{\pi}\left(G, g_{0}(\tau, \varepsilon)\right) d x d \tau \\
& l_{2}(G)=\frac{\omega_{0}}{2 \pi^{2}} \int_{0}^{2 \pi / \omega_{0}} \int_{0}^{\pi}\left(G, g_{1}(\tau, \varepsilon)\right) \cos x d x d \tau .
\end{aligned}
$$

Исследование уравнения (2.79) не вызывает затруднений. В самом деле, из неравенства $\left\|\Pi^{-1}(\varepsilon)\right\|_{\widetilde{E}_{0} \rightarrow \widetilde{E}_{1}} \leqslant M / \varepsilon($ см. (2.74)) и из описанных выше общих свойств функции $\Psi$ вытекает, что правая часть этого уравнения порождает в $\widetilde{E}_{1}$ нелинейный оператор, преобразующий в себя некоторый замкнутый шар не зависящего от $\varepsilon, \mu$ радиуса с центром в нуле и являющийся сжимающим (с константой сжатия порядка $\sqrt{\mu}$ ). Таким образом, из (2.79) однозначно определяется функция

$$
h=h_{*}\left(\tau, x, \varepsilon, \mu, \eta_{0}, \widetilde{\delta}\right) \in \widetilde{E}_{1}: \quad\left\|h_{*}\right\|_{E_{1}}+\left\|\frac{\partial h_{*}}{\partial \eta_{0}}\right\|_{E_{1}}+\left\|\frac{\partial h_{*}}{\partial \widetilde{\delta}}\right\|_{E_{1}} \leqslant M,
$$

где, как обычно, буквой $M$ обозначена некоторая универсальная положительная постоянная, точное значение которой несущественно. В данном случае эта постоянная не зависит от $\varepsilon$ и $\mu$, но, вообще говоря, зависит от компактов, на которых меняются параметры $\eta_{0}, \widetilde{\delta}$.

На заключительном этапе занулим сделанные выше поправки к правой части $G$ из $(2.79)$, т.е. обратимся к равенствам $l_{j}(G)=0, j=1,2$, и подставим 
в них функцию (2.80). Получившиеся в итоге уравнения будем рассматривать как систему для отыскания двух имеющихся в запасе свободных параметров $\eta_{0}, \widetilde{\delta}$. Несложный подсчет показывает, что упомянутые уравнения приводятся к виду

$$
\beta_{1}(\varepsilon) \eta_{0}+\beta_{2}(\varepsilon) \eta_{0}^{3}+O(\sqrt{\mu})=0, \quad \widetilde{\delta}=O(\sqrt{\mu}),
$$

где $\beta_{1}(\varepsilon), \beta_{2}(\varepsilon)$ - коэффициенты (2.63), (2.64). Заметим, далее, что остатки в $(2.81)$ имеют выписанный порядок малости равномерно по $\varepsilon$ и, более того, сохраняются при дифференцировании по $\eta_{0}, \widetilde{\delta}$ (последнее - следствие гладкости по $\eta_{0}, \widetilde{\delta}$ правой части $G$ в (2.79) и функции (2.80)). А отсюда, в свою очередь, следует, что система (2.81) допускает решение вида

$$
\eta_{0}(\varepsilon, \mu)=\sqrt{-\frac{\beta_{1}(\varepsilon)}{\beta_{2}(\varepsilon)}}+O(\sqrt{\mu}), \quad \widetilde{\delta}(\varepsilon, \mu)=O(\sqrt{\mu}) .
$$

И наконец, подставляя соотношения (2.82) вместе с функцией (2.80) в выражения (2.77), убеждаемся в существовании требуемых пар функций (2.75) с асимптотикой (2.76). Лемма 2.4 доказана.

2.4. Исследование устойчивости. Из установленной выше леммы 2.4 вытекает, что исходная задача (2.12) при независимо меняющихся малых параметрах $\varepsilon, \mu>0$ имеет два пространственно неоднородных цикла:

$$
u=u_{*}(\tau, \varepsilon)+\sqrt{\varepsilon} v_{ \pm}(\tau, x, \varepsilon, \mu), \quad \frac{d \tau}{d t}=\sigma(\varepsilon)(1+\varepsilon \delta(\varepsilon, \mu)) .
$$

Ясно также, что поскольку эти циклы переходят друг в друга при замене $\pi-x \rightarrow x$, то свойства их устойчивости одинаковы. Поэтому ниже ограничимся анализом устойчивости только одного их них, а именно, возьмем цикл (2.83), которому соответствует знак + .

Выполним в системе $(2.12)$ замену времени $\tau=\sigma(\varepsilon)(1+\varepsilon \delta(\varepsilon, \mu)) t$, а после этого линеаризуем ее на упомянутом выше цикле (2.83). В результате приходим к линейной краевой задаче

$$
\Pi(\varepsilon, \mu) h \equiv \Pi(\varepsilon) h+\varepsilon \Sigma(\varepsilon, \mu) h=0,\left.\quad \frac{\partial h}{\partial x}\right|_{x=0}=\left.\frac{\partial h}{\partial x}\right|_{x=\pi}=0 .
$$

Здесь $\Pi(\varepsilon)$ - дифференциальный оператор из (2.42), а оператор $\Sigma$ задается равенством

$$
\begin{aligned}
\Sigma(\varepsilon, \mu) h= & \frac{1}{1+\varepsilon \delta(\varepsilon, \mu)}[\delta(\varepsilon, \mu) A(\tau, \varepsilon) h-B(\tau, x, \varepsilon, \mu) h \\
& \left.+(\delta(\varepsilon, \mu) \nu(\varepsilon)+\mu) D_{0} \frac{\partial^{2} h}{\partial x^{2}}\right]
\end{aligned}
$$

где

$$
B(\tau, x, \varepsilon, \mu)=\left.\Phi_{v}^{\prime}(\tau, v, \varepsilon)\right|_{v=v_{+}(\tau, x, \varepsilon, \mu)},
$$


$\Phi(\tau, v, \varepsilon)$ - функция (2.36). Привлекая, далее, тейлоровское разложение (2.38) и асимптотическое равенство для $v_{+}$(см. (2.76)), убеждаемся, что матрица $(2.86)$ допускает представление вида

$$
B(\tau, x, \varepsilon, \mu)=\sqrt{\mu} B_{1}(\tau, x, \varepsilon)+\mu B_{2}(\tau, x, \varepsilon, \mu),
$$

где

$$
\begin{aligned}
B_{1}(\tau, x, \varepsilon) h & =2 \Phi_{2}\left(\tau, v_{0, *}, h, \varepsilon\right), \\
B_{2}(\tau, x, \varepsilon, 0) h & =2 \Phi_{2}\left(\tau, v_{1, *}, h, \varepsilon\right)+3 \Phi_{3}\left(\tau, v_{0, *}, v_{0, *}, h, \varepsilon\right) .
\end{aligned}
$$

Для выявления свойств устойчивости решений получившейся краевой задачи (2.84) сделаем сначала в ней замену

$$
h \exp \left(\varepsilon \lambda_{0} \tau\right) \rightarrow h
$$

где постоянная $\lambda_{0}>0$ фиксирована и достаточно мала. А именно, считаем, что гурвицевы все матрицы $C_{k}\left(\nu_{*}\right)+\lambda_{0} I, k \geqslant 2, \operatorname{uet}\left(C_{k}\left(\nu_{*}\right)+\lambda_{0} I\right)<0$ при $k=0,1$. Из отмеченных в п. 1.3 спектральных свойств матриц (1.24) при $\nu=\nu_{*}$ следует, что именно такая ситуация и реализуется при достаточно малых $\lambda_{0}>0$.

Одним из мотивов для указанного способа выбора $\lambda_{0}$ служит тот факт, что в этом случае в силу лемм 2.1, 2.2 оператор $\Pi(\varepsilon)-\varepsilon \lambda_{0} I$ имеет ограниченный обратный, действующий из $E_{0}$ в $E_{1}$ и допускающий оценку

$$
\left\|\left(\Pi(\varepsilon)-\varepsilon \lambda_{0} I\right)^{-1}\right\|_{E_{0} \rightarrow E_{1}} \leqslant \frac{M_{1}}{\varepsilon}
$$

с не зависящей от $\varepsilon$ постоянной $M_{1}>0$. Далее, свойство обратимости $\Pi(\varepsilon)-\varepsilon \lambda_{0} I$ вместе с вытекающей из (2.85)-(2.88) оценкой

$$
\|\Sigma(\varepsilon, \mu)\|_{E_{1} \rightarrow E_{0}} \leqslant M_{2} \sqrt{\mu}, \quad M_{2}=\text { const }>0
$$

приводит к выводу, что оператор П $(\varepsilon, \mu)-\varepsilon \lambda_{0} I$ также является обратимым и для него справедливо аналогичное (2.90) неравенство

$$
\left\|\left(\Pi(\varepsilon, \mu)-\varepsilon \lambda_{0} I\right)^{-1}\right\|_{E_{0} \rightarrow E_{1}} \leqslant \frac{M}{\varepsilon},
$$

где постоянная $M>0$ не зависит также и от $\mu$.

Последующий анализ опирается на теорию экспоненциальной дихотомии решений линейных параболических уравнений, изложенную в статье [13]. Результаты этой статьи позволяют заключить, что в силу равномерной по $\mu$ регулярности оператора $\Pi(\varepsilon, \mu)-\varepsilon \lambda_{0} I$ (см. (2.92)) свойства устойчивости соответствующей краевой задачи

$$
\left(\Pi(\varepsilon, \mu)-\varepsilon \lambda_{0} I\right) h=0,\left.\quad \frac{\partial h}{\partial x}\right|_{x=0}=\left.\frac{\partial h}{\partial x}\right|_{x=\pi}=0
$$

одинаковы при всех достаточно малых $\mu$ вплоть до значения $\mu=0$. В случае же $\mu=0$ у нее имеется экспоненциально устойчивое (с показателем экспоненты 
порядка $\varepsilon$ ) двумерное инвариантное подпространство решений, базис в котором образуют функции $h_{0}(\tau, \varepsilon) \exp \left(\varepsilon \lambda_{0} \tau\right), h_{1}(\tau, \varepsilon) \exp \left(\varepsilon \lambda_{0} \tau\right) \cos x$. А отсюда и из вышесказанного следует, что аналогичное устойчивое двумерное подпространство решений сохраняется при всех достаточно малых $\varepsilon, \mu>0$ не только у задачи (2.93), но и у исходной системы (2.84), так как последняя получается из (2.93) в результате обращения замены (2.89).

Итак, проблема устойчивости решений краевой задачи (2.84) сводится к анализу ее поведения на двумерном устойчивом инвариантном подпространстве. Как будет показано ниже, базисом в этом подпространстве являются столбцы матрицы-строки размера $N \times 2$ вида

$$
h=\left[e_{0}(\tau, x, \varepsilon, \mu), e_{1}(\tau, x, \varepsilon, \mu)\right] \exp [\varepsilon \mathscr{D}(\varepsilon, \mu) \tau],
$$

$e_{0}(\tau, x, \varepsilon, \mu), e_{0}(\tau, x, \varepsilon, 0)=h_{0}(\tau, \varepsilon)$, и $e_{1}(\tau, x, \varepsilon, \mu), e_{1}(\tau, x, \varepsilon, 0)=h_{1}(\tau, \varepsilon) \cos x,-$ некоторые $2 \pi / \omega_{0}$-периодические по $\tau$ вектор-функции, подлежащие определению вместе с элементами $d_{j}(\varepsilon, \mu), d_{j}(\varepsilon, 0) \equiv 0, j=1,2$, матрицы

$$
\mathscr{D}(\varepsilon, \mu)=\left(\begin{array}{ll}
0 & d_{1}(\varepsilon, \mu) \\
0 & d_{2}(\varepsilon, \mu)
\end{array}\right) .
$$

Следует отметить, впрочем, что на самом деле функция $e_{0}$ нам уже известна, так как краевая задача (2.84) допускает, очевидно, периодическое решение

$$
\begin{aligned}
e_{0} & =h_{0}(\tau, \varepsilon)+\frac{\partial}{\partial \tau} v_{+}(\tau, x, \varepsilon, \mu) \\
& =h_{0}(\tau, \varepsilon)+\sqrt{\mu} \eta_{0}(\varepsilon) \frac{\partial h_{1}}{\partial \tau}(\tau, \varepsilon) \cos x+O(\mu),
\end{aligned}
$$

где, напомним, $\eta_{0}(\varepsilon)$ - амплитуда (2.65). Что же касается функции $e_{1}$ и элементов матрицы (2.95), то после подстановки соотношений $(2.94),(2.96)$ в (2.84) для них получаем краевую задачу

$$
\Pi(\varepsilon) e_{1}+\varepsilon \Sigma(\varepsilon, \mu) e_{1}+\varepsilon \sigma(\varepsilon)\left(d_{1} e_{0}+d_{2} e_{1}\right)=0,\left.\quad \frac{\partial e_{1}}{\partial x}\right|_{x=0}=\left.\frac{\partial e_{1}}{\partial x}\right|_{x=\pi}=0 .
$$

Как и при построении асимптотики циклов (2.83), изложим сначала алгоритмическую часть проблемы нахождения $e_{1}, d_{1}, d_{2}$. С этой целью подставим в (2.97) ряды

$$
\begin{gathered}
e_{1}=e_{1,0}(\tau, x, \varepsilon)+\sqrt{\mu} e_{1,1}(\tau, x, \varepsilon)+\mu e_{1,2}(\tau, x, \varepsilon)+\cdots, \\
d_{1}=\sqrt{\mu} d_{1,0}(\varepsilon)+\mu d_{1,1}(\varepsilon)+\cdots, \quad d_{2}=\mu d_{2,0}(\varepsilon)+\mu^{3 / 2} d_{2,1}(\varepsilon)+\cdots,
\end{gathered}
$$

где $e_{1,0}=h_{1}(\tau, \varepsilon) \cos x$, и будем последовательно приравнивать коэффициенты при одинаковых степенях $\mu$. В результате для $e_{1,1}, e_{1,2}$ и т.д. получаются линейные неоднородные краевые задачи вида

$$
\Pi(\varepsilon) e_{1, j}=\varepsilon f_{j}(\tau, x, \varepsilon),\left.\quad \frac{\partial e_{1, j}}{\partial x}\right|_{x=0}=\left.\frac{\partial e_{1, j}}{\partial x}\right|_{x=\pi}=0, \quad j \geqslant 1
$$


а коэффициенты разложений для $d_{1}, d_{2}$ определяются из условий их разрешимости в классе $2 \pi / \omega_{0}$-периодических функций, т.е. из аналогичных $(2.44)$ равенств.

Действуя по описанному выше правилу, на первом шаге алгоритма приходим к краевой задаче (2.99) при $j=1$ с неоднородностью $f_{1}$, задающейся аналогичной (2.43) формулой

$$
f_{1}=2 \eta_{0}(\varepsilon) \Phi_{2}\left(\tau, h_{1}(\tau, \varepsilon), h_{1}(\tau, \varepsilon), \varepsilon\right) \cos ^{2} x-\sigma(\varepsilon) d_{1,0}(\varepsilon) h_{0}(\tau, \varepsilon) .
$$

Поэтому здесь, опуская технические детали, приведем сразу окончательный результат:

$$
d_{1,0}(\varepsilon)=2 \eta_{0}(\varepsilon) \delta_{1,0}(\varepsilon), \quad e_{1,1}=2 \eta_{0}(\varepsilon)\left(v_{1,0}(\tau, \varepsilon)+v_{1,2}(\tau, \varepsilon) \cos 2 x\right),
$$

где функции $\delta_{1,0}(\varepsilon), v_{1,0}(\tau, \varepsilon), v_{1,2}(\tau, \varepsilon)$ те же самые, что и в $(2.45),(2.47)$.

Второй шаг алгоритма связан с рассмотрением краевой задачи (2.99) при $j=2$, в которой

$$
\begin{aligned}
f_{2}= & f_{2}^{0}(\tau, x, \varepsilon)-\sigma(\varepsilon)\left(d_{1,1} h_{0}(\tau, \varepsilon)+d_{2,0} h_{1}(\tau, \varepsilon) \cos x\right) \\
f_{2}^{0}=- & \sigma(\varepsilon) \delta_{1}(\varepsilon) \frac{\partial e_{1,0}}{\partial \tau}-D_{0} \frac{\partial^{2} e_{1,0}}{\partial x^{2}}-\sigma(\varepsilon) d_{1,0}(\varepsilon) \eta_{0}(\varepsilon) \frac{\partial h_{1}}{\partial \tau}(\tau, \varepsilon) \cos x \\
& \quad+B_{1}(\tau, x, \varepsilon) e_{1,1}+B_{2}(\tau, x, \varepsilon, 0) e_{1,0}
\end{aligned}
$$

Привлекая, далее, формулы (2.88), (2.100), замечаем, что функция (2.102) получается при формальном дифференцировании правой части из (2.61) по переменной $\eta_{0}$ и последующей подстановке $\eta_{0}=\eta_{0}(\varepsilon)$, где, напомним еще раз, $\eta_{0}(\varepsilon)$ - амплитуда (2.65). А отсюда и из (2.101) автоматически следует, что

$$
d_{1,1}(\varepsilon) \equiv 0, \quad \sigma(\varepsilon) d_{2,0}(\varepsilon)=\beta_{1}(\varepsilon)+3 \beta_{2}(\varepsilon) \eta_{0}^{2}(\varepsilon)=-2 \beta_{1}(\varepsilon)<0,
$$

где $\beta_{1}(\varepsilon), \beta_{2}(\varepsilon)$ - коэффициенты $(2.63),(2.64)$.

Так как знак элемента $d_{2}(\varepsilon, \mu)$ матрицы $(2.95)$ вполне определяется по уже найденной поправке $d_{2,0}(\varepsilon)$ (см. (2.103)), то в дальнейшей реализации алгоритма нахождения коэффициентов рядов (2.98) нет необходимости. Поэтому перейдем к соответствующему строгому результату.

Лемма 2.5. Найдутся такие достаточно малье $\varepsilon_{0}, \mu_{0}>0$, что при всех $0<\varepsilon \leqslant \varepsilon_{0}, 0<\mu \leqslant \mu_{0}$ существует единственная тройка функций

$$
\begin{aligned}
& \left(d_{1}(\varepsilon, \mu), d_{2}(\varepsilon, \mu), e_{1}(\tau, x, \varepsilon, \mu)\right): \quad d_{1}(\varepsilon, 0) \equiv d_{2}(\varepsilon, 0) \equiv 0, \\
& e_{1}(\tau, x, \varepsilon, 0)=h_{1}(\tau, \varepsilon) \cos x, \quad e_{1}\left(\tau+2 \pi / \omega_{0}, x, \varepsilon, \mu\right) \equiv e_{1}(\tau, x, \varepsilon, \mu),
\end{aligned}
$$

обращающих краевую задачу (2.97) в тождество и допускающих асимптотические представления

$$
\begin{gathered}
d_{1}(\varepsilon, \mu)=\sqrt{\mu} d_{1,0}(\varepsilon)+\mu^{3 / 2} \widetilde{d}_{1, *}(\varepsilon, \mu), \quad d_{2}(\varepsilon, \mu)=\mu d_{2,0}(\varepsilon)+\mu^{3 / 2} \widetilde{d}_{2, *}(\varepsilon, \mu), \\
e_{1}(\tau, x, \varepsilon, \mu)=e_{1,0}(\tau, x, \varepsilon)+\sqrt{\mu} e_{1,1}(\tau, x, \varepsilon)+\mu \widetilde{h}_{*}(\tau, x, \varepsilon, \mu), \quad \widetilde{h}_{*} \in \widetilde{E}_{1},
\end{gathered}
$$

где $\left|\widetilde{d}_{j, *}(\varepsilon, \mu)\right| \leqslant M_{j}, j=1,2,\left\|\widetilde{h}_{*}\right\|_{E_{1}} \leqslant M_{3}$, а постоянные $M_{j}>0, j=1,2,3$, не зависят от $\varepsilon, \mu$. 
ДокАзАтельство. Обоснование сформулированного утверждения проводится в точности по той же схеме, что и доказательство леммы 2.4. Поэтому ниже опустим некоторые технические детали и сосредоточимся лишь на характерных особенностях.

Подставим в (2.97) соотношения

$$
\begin{aligned}
& d_{1}=\sqrt{\mu} d_{1,0}(\varepsilon)+\mu d_{1, *}, \quad d_{2}=\mu d_{2, *}, \\
& e_{1}=e_{1,0}(\tau, x, \varepsilon)+\sqrt{\mu} e_{1,1}(\tau, x, \varepsilon)+\mu \widetilde{h}_{*},
\end{aligned}
$$

где $d_{1, *}, d_{2, *}$ - пока произвольные параметры, пробегающие некоторые фиксированные компактные множества, $\widetilde{h}_{*}-$ подлежащая определению функция из пространства $\widetilde{E}_{1}$. В результате для $\widetilde{h}_{*}$ получаем линейную неоднородную краевую задачу вида

$$
\begin{gathered}
\Pi(\varepsilon) \tilde{h}_{*}=-\varepsilon \mu \sigma(\varepsilon) d_{2, *} \widetilde{h}_{*}-\varepsilon \Sigma(\varepsilon, \mu) \widetilde{h}_{*}+\varepsilon f_{2}^{0}(\tau, x, \varepsilon)+\sqrt{\mu} \varepsilon \widetilde{f}\left(\tau, x, \varepsilon, \mu, d_{1, *}, d_{2, *}\right) \\
-\varepsilon \sigma(\varepsilon)\left(d_{1, *} h_{0}(\tau, \varepsilon)+d_{2, *} h_{1}(\tau, \varepsilon) \cos x\right), \\
\left.\frac{\partial \widetilde{h}_{*}}{\partial x}\right|_{x=0}=\left.\frac{\partial \widetilde{h}_{*}}{\partial x}\right|_{x=\pi}=0,
\end{gathered}
$$

где $f_{2}^{0}$ - неоднородность (2.102), а вектор-функция $\widetilde{f}$ и ее первые производные по $d_{1, *}, d_{2, *}$ ограничены равномерно по $\varepsilon, \mu$ в метрике пространства $E_{0}$.

Анализ задачи (2.107) практически дословно повторяет соответствующее место из доказательства леммы 2.4. Действительно, обозначим через $\varepsilon G$ правую часть уравнения из (2.107) и рассмотрим сначала аналогичное (2.79) вспомогательное интегральное уравнение в пространстве $\widetilde{E}_{1}$. Применяя, далее, к упомянутому уравнению принцип сжимающих отображений (справедливость которого вытекает из явного вида $G$ и, в частности, из оценки $(2.91))$, однозначно определяем его решение $\widetilde{h}_{*} \in \widetilde{E}_{1}$. После этого, зануляя сделанные ранее поправки к $G$, находим оставшиеся в запасе параметры $d_{1, *}, d_{2, *}$, для которых на этом пути получаются равномерные по $\varepsilon$ асимптотические равенства

$$
d_{1, *}=O(\sqrt{\mu}), \quad d_{2, *}=-\frac{2 \beta_{1}(\varepsilon)}{\sigma(\varepsilon)}+O(\sqrt{\mu}) .
$$

И наконец, подставляя (2.108) в (2.106), убеждаемся в существовании и единственности требуемых функций (2.104) с асимптотикой (2.105). Лемма 2.5 доказана.

Подведем некоторый итог. Из проделанного в данном пункте линейного анализа вытекает, что краевая задача (2.84) имеет два так называемых критических характеристических показателя, обращающихся в нуль при $\mu=0$. Один из этих показателей в точности нулевой, а другой совпадает с элементом $d_{2}(\varepsilon, \mu)$ матрицы $(2.95)$ и в силу $(2.103)$ является отрицательным при $\mu>0$. Остальные же ее показатели равномерно по $\mu$ находятся в комплексной полуплоскости вида $\{\lambda: \operatorname{Re} \lambda \leqslant-\varepsilon \gamma\}, \gamma=$ const $>0$. А это означает, что пространственно неоднородные циклы (2.83) краевой задачи (2.12) экспоненциально орбитально устойчивы при всех достаточно малых $\varepsilon, \mu>0$, и, следовательно, при 
замене условия (2.13) на равенство (2.14) утверждение теоремы 2.1 полностью обосновано.

2.5. Завершение доказательства. В этом пункте поясняются причины справедливости теоремы 2.1 в общем случае, когда мы не требуем выполнения равенства (2.14), но предполагаем, что имеют место условия нерезонансности (2.13).

Как известно (см., например, [14]), при условиях (2.13) существует замена переменных вида $u \rightarrow u+\Delta(u, u)$ (где $\Delta(u, u)$ - симметричная квадратичная форма), “убивающая" в системе (1.8) при $\varepsilon=0$ квадратичное слагаемое $F_{2}(u, u)$ (см. (1.3)). Выполним, далее, упомянутую замену в краевой задаче (2.12). В результате она примет вид

$$
\begin{gathered}
\frac{\partial u}{\partial t}=\varepsilon D(\varepsilon, \mu) \frac{\partial^{2} u}{\partial x^{2}}+A(\varepsilon) u+\widetilde{F}(u, \varepsilon)+\varepsilon \widetilde{G}\left(u, \frac{\partial u}{\partial x}, \frac{\partial^{2} u}{\partial x^{2}}, \varepsilon, \mu\right), \\
\left.\frac{\partial u}{\partial x}\right|_{x=0}=\left.\frac{\partial u}{\partial x}\right|_{x=\pi}=0,
\end{gathered}
$$

где матрица $A(\varepsilon)$ остается прежней, а для нелинейности $\widetilde{F}(u, \varepsilon)$ сохраняются общие свойства (1.3), но теперь тейлоровское разложение в нуле функции $\widetilde{F}(u, 0)$ начинается с кубического члена. Что же касается нового слагаемого $\varepsilon \widetilde{G}$, то вектор-функция $\widetilde{G}(u, v, w, \varepsilon, \mu)$ в нем задается равенством

$$
\begin{aligned}
\widetilde{G}=[ & \left.\left(I+\Delta^{\prime}(u)\right)^{-1}-I\right] D(\varepsilon, \mu) w+\left(I+\Delta^{\prime}(u)\right)^{-1}[2 D(\varepsilon, \mu) \Delta(v, v) \\
& \left.+D(\varepsilon, \mu) \Delta^{\prime}(u) w\right],
\end{aligned}
$$

где матрица $\Delta^{\prime}(u)$ определяется по правилу $\Delta^{\prime}(u) h=2 \Delta(u, h), h \in \mathbb{R}^{N}$.

Переход от исходной краевой задачи (2.12) к (2.109) решает проблему обоснования теоремы 2.1, так как для получившейся задачи (2.109) утверждения лемм 2.1-2.5 остаются в силе. Действительно, из явного вида (2.110) векторфункции $\widetilde{G}$ вытекает, что ее тейлоровское разложение по переменным $(u, v, w)$ в точке $(0,0,0)$ начинается с квадратичных слагаемых, а значит, задача $(2.109)$ отличается от (2.12) только нелинейными добавками, пропорциональными $\varepsilon$.

Из проделанного в пп. 2.1-2.4 анализа следует, что такого типа добавки не могут повлиять на структуру всех встречающихся выше асимптотических формул. Не создает дополнительных трудностей и факт зависимости $\widetilde{G}$ от $\partial^{2} u / \partial x^{2}, \partial u / \partial x$, поскольку для оператора $\Pi^{-1}(\varepsilon)$ нами была установлена коэрцитивная оценка вида (2.74). По этой же причине сохраняются с незначительными изменениями все основные конструкции, используемые при обосновании лемм 2.1-2.5. Например, фигурирующие в (2.78) и (2.84) операторы $\Psi$ и $\Sigma$ в случае задачи (2.109) будут зависеть еще и от $\partial h / \partial x$. Однако общие свойства данных операторов, которые, собственно, и требуются при доказательстве лемм 2.4 и 2.5 , от этого не изменятся.

Теорема 2.1 полностью доказана. 


\section{$\S 3$. Заключение}

Следует отметить, что практически все результаты по бифуркациям в параболических системах с малой диффузией так или иначе связаны с методом квазинормальных форм. А это значит, что проблемы возможных обобщений и расширений данного метода весьма актуальны. Одна из таких проблем решена в настоящей статье.

Действительно, в первую очередь обратим внимание на тот факт, что пространственно неоднородные циклы краевой задачи (2.12), доставляемые теоремами 1.3 и 2.1, совпадают. В предположении противного фиксируем в исходной задаче (2.12) параметры $\varepsilon$ и $\mu$ в соответствии с теоремой 1.3. Тогда, очевидно, она будет удовлетворять условиям теорем 1.3 и 2.1 одновременно, гарантирующих существование у нее четырех различных пространственно неоднородных циклов. Далее, заметим, что в силу формул $(2.34),(2.40),(2.41)$ циклы, о которых говорится в теореме 2.1 , имеют амплитуды порядка $\sqrt{\varepsilon}$ и располагаются в $\sqrt{\varepsilon \mu}$-окрестности однородного цикла, т.е. обладают теми же свойствами, что и циклы из теоремы 1.3. Следовательно, все эти четыре цикла должны “ловиться" с помощью метода квазинормальных форм, т.е. в краевой задаче $(1.15),(1.16)$ им должны соответствовать четыре различных пространственно неоднородных автомодельных цикла. Однако, как показано в п. 1.3, квазинормальная форма $(1.15),(1.16)$ при условиях (1.26) в окрестности однородного цикла (1.22) имеет только два пространственно неоднородных цикла вида (1.27). Получаем противоречие с исходной посылкой.

Суммируя все вышеизложенное, приходим к выводу, что как в краевой задаче (1.1), так и в квазинормальной форме (1.15), (1.16) при условии (1.19) и при уменьшении параметра $\nu$ (точнее говоря, при прохождении его через критические значения $\nu(\varepsilon)$ и $\nu_{*}$ соответственно) происходит одна и та же бифуркация типа вилки: однородный цикл теряет устойчивость и порождает два устойчивых пространственно неоднородных периодических решения. А это значит, что нам удалось несколько расширить границы применимости метода квазинормальных форм и показать, что соответствие между автомодельными циклами квазинормальной формы (1.15), (1.16) и циклами вида $(1.11),(1.12)$ исходной системы (1.1) имеет место не только в грубом случае (см. теоремы $1.1,1.3)$, но и в момент бифуркации.

Скажем несколько слов о роли условий нерезонансности (2.13). С одной стороны, они не являются обременительными, так как характеризуют некоторую общность положения и заведомо выполняются при $N=2$ и $N=3$ (в этих двух случаях собственные значения матрицы $A_{0}$ исчерпываются наборами $\lambda= \pm i \omega_{0}$ и $\lambda= \pm i \omega_{0}, \lambda=\lambda_{0}<0$ соответственно, а значит, резонансы второго порядка здесь не возникают). С другой стороны, в рамках избранной нами схемы исследования отказ от неравенств (2.13) не представляется возможным. Действительно, при нарушении данных условий не удается избавиться от квадратичной нелинейности в (1.3) и в силу этого сталкиваемся, например, со следующей проблемой: множитель $\varepsilon \sqrt{\mu}$ перед функцией $\Psi$ в уравнении $(2.78)$ 
заменяется на $\sqrt{\varepsilon \mu}$. Подобная замена приводит к тому, что оператор, порождаемый в пространстве $\widetilde{E}_{1}$ правой частью уравнения $(2.79)$, перестает быть сжимающим, так как теперь он будет удовлетворять условию Липшица с константой порядка $\sqrt{\mu / \varepsilon}$, а не $\sqrt{\mu}$.

Хотя вопрос о справедливости теоремы 2.1 без условий (2.13) пока остается открытым, весьма правдоподобно, что ответ на него окажется положительным. Косвенным аргументом в пользу этой гипотезы служит тот факт, что от аналогичных условий удалось избавиться в теоремах 1.1 и 1.3 (см. соответствующее место в монографии [6]).

Остановимся на двух возможных обобщениях теоремы 2.1. Первое из них относится к матричному коэффициенту диффузии $D(\varepsilon, \mu)$, а точнее говоря, к вопросу об усложнении его зависимости от параметров $\varepsilon, \mu$. Нетрудно убедиться, что наш основной результат сохраняется при любой достаточно гладко зависящей от $(\varepsilon, \mu) \in\left[0, \varepsilon_{0}\right] \times\left[0, \mu_{0}\right], \varepsilon_{0}, \mu_{0}=$ const $>0$, матрице $D(\varepsilon, \mu)$, удовлетворяющей следующим ограничениям:

1) гурвицевыми являются матрица $-D(0,0)$, а также матрица $A_{0}-z D(0,0)$ при любом $z>0$;

$2)$ выполняется неравенство $\operatorname{Re}(D(0,0) a, b)>0$;

3) справедливо условие (1.20), в котором

$$
x_{0}=-\frac{\operatorname{Im}(D(0,0) a, b)}{\operatorname{Re}(D(0,0) a, b)}
$$

4) гурвицевыми являются все матрицы

$$
C_{k}=-\left(\begin{array}{cc}
\phi_{0}\left(1+i y_{0}\right) & \phi_{0}\left(1+i y_{0}\right) \\
\phi_{0}\left(1-i y_{0}\right) & \phi_{0}\left(1-i y_{0}\right)
\end{array}\right)-\left(\begin{array}{cc}
k^{2}(D(0,0) a, b) & 0 \\
0 & k^{2}(D(0,0) \bar{a}, \bar{b})
\end{array}\right)
$$

при $k \geqslant 2$, а матрица $C_{1}$ имеет нулевое собственное значение;

$5)$ аналогичная (2.15) линейная система

$$
\sigma(\varepsilon) \frac{d h}{d \tau}-[A(\tau, \varepsilon)-\varepsilon D(\varepsilon, 0)] h=0
$$

имеет простой единичный мультипликатор, а остальные ее мультипликаторы по модулю строго меньше единицы;

6) матрица $D_{1}=D_{\mu}^{\prime}(0,0)$ такова, что

$$
\operatorname{Re}\left[\left(D_{1} a, b\right)\left(\gamma_{1}+i \gamma_{2}\right)\left(1+i x_{0}\right)\right]<0,
$$

где, напомним, $\gamma_{1}=1-x_{0} y_{0}, \gamma_{2}=x_{0}+y_{0}$.

Второе обобщение связано с переходом в (2.12) от отрезка к плоской области, например к квадрату $\Omega=\left\{\left(x_{1}, x_{2}\right): 0 \leqslant x_{1} \leqslant \pi, 0 \leqslant x_{2} \leqslant \pi\right\}$. Получающуюся при этом краевую задачу $(\Delta-$ оператор Лапласа)

$$
\frac{\partial u}{\partial t}=\varepsilon D(\varepsilon, \mu) \Delta u+A(\varepsilon) u+F(u, \varepsilon),\left.\quad \frac{\partial u}{\partial x_{1}}\right|_{x_{1}=0, \pi}=\left.\frac{\partial u}{\partial x_{2}}\right|_{x_{2}=0, \pi}=0
$$


следует рассматривать в фазовом пространстве $\stackrel{\circ}{W_{2}^{2}}\left(\Omega ; \mathbb{R}^{N}\right)$, где $\stackrel{\circ}{W_{2}^{2}}$ - замыкание в метрике соболевского пространства $W_{2}^{2}\left([0, \pi] ; \mathbb{R}^{N}\right)$ линеала гладких вектор-функций, удовлетворяющих граничным условиям из (3.1).

Для того чтобы установить в случае задачи (3.1) аналог теоремы 2.1, сначала, предполагая выполненными все условия данной теоремы, проведем бифуркационный анализ соответствующей квазинормальной формы

$$
\begin{gathered}
\frac{\partial \xi}{\partial s}=\left(\nu_{*}-\mu\right)\left(1-i x_{0}\right) \Delta \xi+\left(A_{1} a, b\right) \xi+d|\xi|^{2} \xi \\
\left.\frac{\partial \xi}{\partial x_{1}}\right|_{x_{1}=0, \pi}=\left.\frac{\partial \xi}{\partial x_{2}}\right|_{x_{2}=0, \pi}=0
\end{gathered}
$$

где $0<\mu \ll 1, \nu_{*}>0-$ критическое значение из (1.25). В связи с этим обратим внимание на то, что в окрестности однородного цикла (1.22) краевая задача (3.2) имеет аналогичное (1.45) экспоненциально орбитально устойчивое (в метрике фазового пространства $\left.(\operatorname{Re} \xi, \operatorname{Im} \xi) \in \stackrel{\circ}{W_{2}^{2}}(\Omega) \times \stackrel{\circ}{W_{2}^{2}}(\Omega)\right)$ трехмерное инвариантное многообразие

$$
\xi=\xi_{0}\left[1+\eta_{1}\left(\gamma_{1}+i \gamma_{2}\right) \cos x_{1}+\eta_{2}\left(\gamma_{1}+i \gamma_{2}\right) \cos x_{2}+H\left(\eta_{1}, \eta_{2}, x_{1}, x_{2}, \mu\right)\right] \exp (i \psi)
$$

Здесь $\eta_{1}, \eta_{2}, \psi$ - вещественные параметры на многообразии, причем $\left|\eta_{j}\right| \leqslant q$, $j=1,2$, где $q>0$ достаточно мало, а достаточно гладкая по совокупности переменных комплекснозначная функция $H$ такова, что

$$
\begin{gathered}
H\left(0,0, x_{1}, x_{2}, \mu\right) \equiv \frac{\partial H}{\partial \eta_{1}}\left(0,0, x_{1}, x_{2}, 0\right) \equiv \frac{\partial H}{\partial \eta_{2}}\left(0,0, x_{1}, x_{2}, 0\right) \equiv 0, \\
\left.\frac{\partial H}{\partial x_{1}}\right|_{x_{1}=0, \pi}=\left.\frac{\partial H}{\partial x_{2}}\right|_{x_{2}=0, \pi}=0, \\
\operatorname{Im}\left[\left(1-i y_{0}\right) \int_{0}^{\pi} \int_{0}^{\pi} H\left(\eta_{1}, \eta_{2}, x_{1}, x_{2}, \mu\right) d x_{1} d x_{2}\right]=0 .
\end{gathered}
$$

Система уравнений на многообразии (3.3) впервые была выписана в статье [15]. Как и аналогичная ей система (1.46), она имеет треугольный вид

$$
\begin{aligned}
\frac{d \eta_{1}}{d s} & =\varkappa_{1} \mu \eta_{1}+\varkappa_{2}\left[Q \eta_{1}^{2}+P \eta_{2}^{2}\right] \eta_{1}+\Phi_{1}\left(\eta_{1}, \eta_{2}, \mu\right), \\
\frac{d \eta_{2}}{d s} & =\varkappa_{1} \mu \eta_{2}+\varkappa_{2}\left[P \eta_{1}^{2}+Q \eta_{2}^{2}\right] \eta_{2}+\Phi_{2}\left(\eta_{1}, \eta_{2}, \mu\right), \\
\frac{d \psi}{d s} & =\omega_{0} \alpha_{0}-\phi_{0}\left(1+y_{0}^{2}\right) \gamma_{1} \gamma_{2}\left(\eta_{1}^{2}+\eta_{2}^{2}\right)+\Phi_{3}\left(\eta_{1}, \eta_{2}, \mu\right),
\end{aligned}
$$

где $\varkappa_{1}=\left(1+x_{0}^{2}\right)\left(x_{0} y_{0}-1\right) /\left(\gamma_{2} x_{0}-\gamma_{1}\right), \varkappa_{2}=\phi_{0} /\left(\gamma_{2} x_{0}-\gamma_{1}\right), Q=Q\left(x_{0}, y_{0}\right)-$ коэффициент (1.40), $P=P\left(x_{0}, y_{0}\right)$ задается равенством

$$
P\left(x_{0}, y_{0}\right)=6 \gamma_{1}^{4}-4 \gamma_{1}^{2} \gamma_{2}^{2}+2 \gamma_{1}^{3} \gamma_{2} y_{0}
$$


а функции $\Phi_{j}, j=1,2,3$, достаточно гладко зависят от $\eta_{1}, \eta_{2}, \mu$ и обладают следующими свойствами:

$$
\begin{gathered}
\Phi_{j}(0,0, \mu) \equiv 0, \quad \frac{\partial \Phi_{j}}{\partial \eta_{k}}(0,0,0)=\frac{\partial^{2} \Phi_{j}}{\partial \eta_{k} \partial \mu}(0,0,0)=0, \quad j=1,2,3, \quad k=1,2, \\
\Phi_{1}\left(\eta_{1}, \eta_{2}, \mu\right)=\Phi_{2}\left(\eta_{2}, \eta_{1}, \mu\right) .
\end{gathered}
$$

Кроме того, тейлоровские разложения в нуле функций $\Phi_{j}\left(\eta_{1}, \eta_{2}, 0\right), j=1,2$, и $\Phi_{3}\left(\eta_{1}, \eta_{2}, 0\right)$ начинаются с членов четвертого порядка малости и кубических слагаемых соответственно. Добавим еще, что свойство взаимной симметрии $\Phi_{1}$ и $\Phi_{2}$ (см. (3.6)) - следствие инвариантности многообразия (3.3) относительно замен $x_{1} \rightarrow x_{2}, x_{2} \rightarrow x_{1}$ и $\eta_{1} \rightarrow \eta_{2}, \eta_{2} \rightarrow \eta_{1}$.

За бифуркации, происходящие в краевой задаче $(3.2)$ при $0<\mu \ll 1$, отвечает, очевидно, так называемая укороченная нормальная форма для амплитудных переменных

$$
\frac{d \eta_{1}}{d s}=\varkappa_{1} \eta_{1}+\varkappa_{2}\left[Q \eta_{1}^{2}+P \eta_{2}^{2}\right] \eta_{1}, \quad \frac{d \eta_{2}}{d s}=\varkappa_{1} \eta_{2}+\varkappa_{2}\left[P \eta_{1}^{2}+Q \eta_{2}^{2}\right] \eta_{2},
$$

получающаяся из первых двух уравнений системы (3.4) в результате замен $\mu s \rightarrow s, \eta_{j} / \sqrt{\mu} \rightarrow \eta_{j}, j=1,2$, и последующего отбрасывания асимптотически малых по $\mu$ слагаемых. Нетрудно проверить, что, во-первых, система (3.7) оказывается диссипативной, так как в области $\left\{\left(x_{0}, y_{0}\right): x_{0} y_{0}>1\right\}$ выполняется неравенство $Q\left(x_{0}, y_{0}\right)+P\left(x_{0}, y_{0}\right)<0$; во-вторых, она допускает четыре состояния равновесия вида

$$
\left(\eta_{1}, \eta_{2}\right)=\left( \pm \eta_{*}, 0\right), \quad\left(0, \pm \eta_{*}\right): \quad \eta_{*}=\sqrt{-\frac{\varkappa_{1}}{\varkappa_{2} Q}}
$$

и четыре положения равновесия

$$
\left(\eta_{1}, \eta_{2}\right)=\left( \pm \eta_{* *}, \pm \eta_{* *}\right), \quad\left( \pm \eta_{* *}, \mp \eta_{* *}\right): \quad \eta_{* *}=\sqrt{-\frac{\varkappa_{1}}{\varkappa_{2}(Q+P)}},
$$

причем в случае $P\left(x_{0}, y_{0}\right)<Q\left(x_{0}, y_{0}\right)$ устойчивы равновесия $(3.8)$, а неподвижные точки (3.9) дихотомичны. В случае же $P\left(x_{0}, y_{0}\right)>Q\left(x_{0}, y_{0}\right)$ ситуация меняется на противоположную.

Каждому из найденных выше состояний равновесия укороченной нормальной формы (3.7) в исходной задаче (3.2) отвечает автомодельный цикл с аналогичными свойствами устойчивости, причем неподвижным точкам (3.8) соответствуют периодические решения, зависящие только от одной пространственной переменной. Таким образом, в случае квадрата в квазинормальной форме (3.2) при $0<\mu \ll 1$ и при условии

$$
P\left(x_{0}, y_{0}\right) \neq Q\left(x_{0}, y_{0}\right)
$$

от однородного цикла (1.22) ответвляются сразу восемь пространственно неоднородных периодических решений - четыре устойчивых и четыре неустойчивых. 
Возвращаясь к краевой задаче (3.1), отметим, что для нее разработанная нами в 22 общая схема исследования сохраняется без существенных изменений. А это значит, что справедливо следующее утверждение.

Теорема 3.1. Пусть в дополнение к условиям теоремы 2.1 выполнено неравенство (3.10). Тогда можно указать такие $\varepsilon_{0}, \mu_{0}>0$, что при всех $0<\varepsilon \leqslant \varepsilon_{0}$, $0<\mu \leqslant \mu_{0}$ краевая задача (3.1) в некоторой асимптотически малой (порядка $\sqrt{\varepsilon \mu}$ ) окрестности неустойчивого однородного иикла (1.9) имеет восемь пространственно неоднородных периодических по $t$ решений, четыре из которых экспоненциально орбитально устойчивы, а остальные четыре - дихотомичны.

В заключение приведем конкретный пример краевой задачи (1.1), для которой выполняются все перечисленные в $§ 1$ ограничения. В качестве соответствующей точечной модели возьмем "брюсселятор" (см. [8])

$$
\dot{X}=\alpha-(\beta+1) X+X^{2} Y, \quad \dot{Y}=\beta X-X^{2} Y,
$$

где $\alpha, \beta>0$. Как известно (см., например, [2]), при увеличении параметра $\beta$ и при прохождении его через пороговое значение $\alpha^{2}+1$ в системе (3.11) происходит закритическая бифуркация Андронова-Хопфа: состояние равновесия $X=\alpha, Y=\beta / \alpha$ теряет устойчивость, и от него ответвляется устойчивый цикл. Поэтому положим в (3.11) $X=\alpha+u_{1}, Y=\beta / \alpha+u_{2}, \beta=\alpha^{2}+1+\varepsilon$, где $0<\varepsilon \ll 1$. В результате приходим к аналогичной (1.8) системе

$$
\begin{aligned}
& \dot{u}_{1}=\left(\alpha^{2}+\varepsilon\right) u_{1}+\alpha^{2} u_{2}+2 \alpha u_{1} u_{2}+\frac{\alpha^{2}+1+\varepsilon}{\alpha} u_{1}^{2}+u_{1}^{2} u_{2}, \\
& \dot{u}_{2}=-\left(\alpha^{2}+1+\varepsilon\right) u_{1}-\alpha^{2} u_{2}-2 \alpha u_{1} u_{2}-\frac{\alpha^{2}+1+\varepsilon}{\alpha} u_{1}^{2}-u_{1}^{2} u_{2},
\end{aligned}
$$

удовлетворяющей всем необходимым условиям, поскольку здесь

$$
\begin{gathered}
A_{0}=\left(\begin{array}{cc}
\alpha^{2} & \alpha^{2} \\
-\alpha^{2}-1 & -\alpha^{2}
\end{array}\right), \\
\omega_{0}=\alpha, \quad a=(\alpha, i-\alpha)^{T}, \quad b=\frac{1}{2 \alpha}(1+i \alpha, i \alpha)^{T}, \quad \phi_{0}=\frac{1}{2}, \\
\operatorname{Re} d=-\frac{1}{2}\left(\alpha^{2}+2\right)<0, \quad \operatorname{Im} d=-\frac{2 \alpha}{3}\left[\left(\alpha-\frac{1}{\alpha}\right)^{2}+\frac{1}{4}\right] .
\end{gathered}
$$

Перейдем, далее, от (3.12) к распределенной модели

$$
\begin{gathered}
\frac{\partial u_{1}}{\partial t}=\left(\alpha^{2}+\varepsilon\right) u_{1}+\alpha^{2} u_{2}+2 \alpha u_{1} u_{2}+\frac{\alpha^{2}+1+\varepsilon}{\alpha} u_{1}^{2}+u_{1}^{2} u_{2}+\varepsilon d_{1} \frac{\partial^{2} u_{1}}{\partial x^{2}} \\
\frac{\partial u_{2}}{\partial t}=-\left(\alpha^{2}+1+\varepsilon\right) u_{1}-\alpha^{2} u_{2}-2 \alpha u_{1} u_{2}-\frac{\alpha^{2}+1+\varepsilon}{\alpha} u_{1}^{2}-u_{1}^{2} u_{2}+\varepsilon d_{2} \frac{\partial^{2} u_{2}}{\partial x^{2}} \\
\left.\frac{\partial u_{j}}{\partial x}\right|_{x=0}=\left.\frac{\partial u_{j}}{\partial x}\right|_{x=\pi}=0, \quad j=1,2,
\end{gathered}
$$


предполагая, что коэффициенты диффузии $d_{1}, d_{2}>0$ в ней связаны неравенством

$$
d_{1}>d_{2}
$$

Тогда, как нетрудно убедиться, будут выполняться и все требования, относящиеся к матрице $A_{0}-z D, z>0$, где $D=\operatorname{diag}\left\{d_{1}, d_{2}\right\}$. Действительно, условие (1.7) здесь справедливо автоматически, так как в силу (3.13) имеем

$$
(D a, b)=\frac{1}{2}\left[d_{1}+d_{2}+i \alpha\left(d_{2}-d_{1}\right)\right] .
$$

Что же касается гурвицевости этой матрицы, то она эквивалентна условию $z^{2} d_{1} d_{2}+\alpha^{2}\left(d_{1}-d_{2}\right) z+\alpha^{2}>0$, справедливость которого вытекает из (3.15).

Оставшееся ограничение, а именно оценка $x_{0} y_{0}-1>0$, где

$$
x_{0}=-\frac{\operatorname{Im}(D a, b)}{\operatorname{Re}(D a, b)}, \quad y_{0}=\frac{\operatorname{Im} d}{\operatorname{Re} d},
$$

в случае краевой задачи (3.14) принимает вид

$$
\frac{4 \alpha^{2}}{3} \cdot \frac{1 / 4+(\alpha-1 / \alpha)^{2}}{\alpha^{2}+2} \cdot \frac{d_{1}-d_{2}}{d_{1}+d_{2}}>1
$$

А отсюда и из (3.15) заключаем, что неравенство $x_{0} y_{0}-1>0$ заведомо имеет место при всех достаточно больших значениях параметра $\alpha$.

Подводя итог, отметим следующие два факта. Во-первых, предположим, что коэффициенты диффузии $d_{1}, d_{2}$ в (3.14) пропорциональны некоторому параметру $\nu>0$, т.е. $d_{1}=\nu d_{1}^{0}, d_{2}=\nu d_{2}^{0}$, где $d_{1}^{0}, d_{2}^{0}>0$ фиксированы и удовлетворяют условиям (3.15), (3.16). Тогда при $\nu=\nu(\varepsilon)-\mu, 0<\mu \ll 1$, где

$$
\nu(\varepsilon), \nu(0)=\frac{2\left(d_{1}^{0}+d_{2}^{0}\right)}{\left(d_{1}^{0}+d_{2}^{0}\right)^{2}+\alpha^{2}\left(d_{1}^{0}-d_{2}^{0}\right)^{2}}\left(\frac{4 \alpha^{2}}{3} \cdot \frac{1 / 4+(\alpha-1 / \alpha)^{2}}{\alpha^{2}+2} \cdot \frac{d_{1}^{0}-d_{2}^{0}}{d_{1}^{0}+d_{2}^{0}}-1\right)
$$

представляет собой критическое значение, определенное в п. 2.1, для краевой задачи (3.14) справедливо утверждение теоремы 2.1.

Во-вторых, на качественном уровне наши теоретические результаты согласуются с результатами численного счета из монографии [16]. А именно, в работе [16] проведен численный анализ периодических по $t$ решений краевой задачи

$$
\begin{gathered}
\frac{\partial u_{1}}{\partial t}=\alpha-(\beta+1) u_{1}+u_{1}^{2} u_{2}+\frac{d_{1}}{l^{2}} \frac{\partial^{2} u_{1}}{\partial x^{2}}, \quad \frac{\partial u_{2}}{\partial t}=\beta u_{1}-u_{1}^{2} u_{2}+\frac{d_{2}}{l^{2}} \frac{\partial^{2} u_{2}}{\partial x^{2}}, \\
\left.u_{1}\right|_{x=0}=\left.u_{1}\right|_{x=1}=\alpha,\left.\quad u_{2}\right|_{x=0}=\left.u_{2}\right|_{x=1}=\frac{\beta}{\alpha}
\end{gathered}
$$

при фиксированных параметрах $\alpha=2, \beta=5.45, d_{1}=0.008, d_{2}=0.004$ и при увеличении $l$. Было установлено существование таких двух критических значений $l_{1} \approx 0.513$ и $l_{2} \in(1,1.5)$, что при $0<l<l_{1}$ устойчиво состояние равновесия $u_{1} \equiv \alpha, u_{2} \equiv \beta / \alpha$ задачи (3.17), а при $l=l_{1}$ происходит бифуркация Андронова-Хопфа: от указанного состояния равновесия ответвляется устойчивый пространственно симметричный цикл, для компонент $u_{1}(t, x), u_{2}(t, x)$ 
которого выполняются равенства $u_{j}(t, x) \equiv u_{j}(t, 1-x), j=1,2$. Этот цикл сохраняет устойчивость при $l_{1}<l<l_{2}$, а при последующем увеличении параметра $l$ и при прохождении его через критическое значение $l=l_{2}$ претерпевает бифуркацию типа вилки, т.е. становится неустойчивым и порождает пару устойчивых несимметричных циклов, переходящих друг в друга в результате замены $x$ на $1-x$. Для сравнения заметим, что точно такая же бифуркация наблюдается и в краевой задаче (3.14) при $d_{1}=\nu d_{1}^{0}, d_{2}=\nu d_{2}^{0}$, и при прохождении параметра $\nu$ через свое критическое значение $\nu(\varepsilon)$.

Авторы выражают глубокую признательность рецензентам статьи за внимательное ознакомление с рукописью и высказанные конструктивные замечания и соображения, существенно способствовавшие улучшению содержания статьи.

\section{Список литературы}

[1] Дж. Марсден, М. Мак-Кракен, Бифуркаиия рождения иикла и ее приложения, Мир, М., 1980; пер. с англ.: J.E. Marsden, M. McCracken, The Hopf bifurcation and its applications, Springer-Verlag, New York-Heidelberg-Berlin, 1976.

[2] Б. Хэссард, Н. Казаринов, И. Вэн, Теория и приложения бифуркаиии рождения цикла, Мир, М., 1985; пер. с англ.: В.D. Hassard, N.D. Kazarinoff, Y.-H. Wan, Theory and applications of Hopf bifurcation, London Math. Soc. Lecture Note Ser., 41, Cambridge Univ. Press, Cambridge-New York, 1981.

[3] Е.Ф. Мищенко, Ю.С. Колесов, А. Ю. Колесов, Н.Х. Розов, Периодические движения и бифуркачионные прочессы в сингулярно возмущенных системах, Наука, Физматлит, М., 1995; англ. пер.: Е. F. Mishchenko, Yu.S. Kolesov, A. Yu. Kolesov, N. Kh. Rozov, Asymptotic methods in singularly perturbed systems, Monogr. Contemp. Math., Consultants Bureau, New York, 1994.

[4] А. Ю. Колесов, Н.Х. Розов, Е.Ф. Мищенко, Асимптотические методъ исследования периодических решений нелинейных гиперболических уравнений, Тр. МИАН, 222, Наука, М., 1998; англ. пер.: А. Yu. Kolesov, E. F. Mishchenko, N. Kh. Rozov, "Asymptotic methods of investigation of periodic solutions of nonlinear hyperbolic equations", Proc. Steklov Inst. Math., 222:3 (1998), 1-188.

[5] А. Ю. Колесов, Н.Х. Розов, Инвариантные торы нелинейных волновых уравнений, Физматлит, М., 2004.

[6] Е.Ф. Мищенко, В.А. Садовничий, А. Ю. Колесов, Н.Х. Розов, Автоволновые процессы в нелинейных средах с дифбузией, Физматлит, М., 2005.

[7] Н.Н. Боголюбов, Ю.А. Митропольский, Асимптотические методы в теории нелинейных колебаний, 4-е изд., Наука, М., 1974; англ. пер. 2-го изд.: N. N. Bogolyubov, Yu. A. Mitropol'skii, Asymptotic methods in the theory of nonlinear oscillations, Hindustan Publ., Delhi; Gordon and Breach, New York, 1961.

[8] П. Гленсдорф, И. Пригожин, Термодинамическая теория структуры, устойчивости и флуктуаций, Мир, М., 1973; пер. с англ.: P. Glansdorff, I. Prigogine, Thermodynamic theory of structure, stability and fluctuations, Wiley, London, 1971.

[9] А. Ю. Колесов, "Структура окрестности однородного цикла в среде с диффузией”, Изв. АН СССР. Сер. матем., 53:2 (1989), 345-362; англ. пер.: А. Yu. Kolesov, "The structure of a neighborhood of a homogeneous cycle in a medium with diffusion", Math. USSR-Izv., 34:2 (1990), 355-372.

[10] Ю. С. Колесов, Д. И. Швитра, Автоколебания в системах с запаздыванием, Мокслас, Вильнюс, 1979.

[11] А. Ю. Колесов, "Миграционные эффекты в одновидовом биоценозе”, Нелинейнъе колебания и экология, Изд-во ЯрГУ, Ярославль, 1984, 34-61. 
[12] Ю.С. Колесов, В.В.Майоров, "Новый метод исследования устойчивости решений линейных дифференциальных уравнений с близкими к постоянным почти периодическими коэффициентами", Дифферени. уравнения, 10 (1974), 1778-1788; англ. пер.: Ju. S. Kolesov, V. V. Maiorov, "A new method of investigating the stability of solutions of linear differential equations with almost-periodic coefficients that are almost constant", Differential Equations, 10 (1976), 1363-1370.

[13] Ю. С. Колесов, "Об устойчивости решений линейных дифференциальных уравнений параболического типа с почти периодическими коэффициентами”, Tp. MMO, 36 (1978), 3-27; англ. пер.: Ju. S. Kolesov, "On the stability of solutions of linear differential equations of parabolic type with almost periodic coefficients", Trans. Moscow Math. Soc., 36 (1979), 1-25.

[14] Л. П. Шильников, А. Л. Шильников, Д. В. Тураев, Л. Чуа, Методъ качественной теории в нелинейной динамике. Часть 1, Институт компьютерных исследований, М., Ижевск, 2004; пер. с англ.: L. P. Shilnikov, A. L. Shilnikov, D. V. Turaev, L. O. Chua, Methods of qualitative theory in nonlinear dynamics. Part I, World Sci. Ser. Nonlinear Sci. Ser. A Monogr. Treatises, 4, World Sci. Publ., River Edge, NJ, 1998.

[15] С. Д. Глызин, А. Ю. Колесов, "Установившиеся режимы уравнения Хатчинсона с малой диффузией в случае квадрата", Качественные методы исследования операторных уравнений, Изд-во ЯрГУ, Ярославль, 1988, 44-54.

[16] М. Холодниок, А. Клич, М. Кубичек, М. Марек, Методы анализа нелинейных динамических моделей, Мир, М., 1991.

А. Ю. Колесов (А. Үu. Kolesov)

Ярославский государственный

университет им. П. Г. Демидова

E-mail: kolesov@uniyar.ac.ru

Н. Х. Розов (N. Kh. Rozov)

Московский государственный

университет им. М. В. Ломоносова

E-mail: rozov@rozov.mccme.ru

\section{В. А. Садовничий (V. A. Sadovnichiř)}

Московский государственный университет им. М. В. Ломоносова

E-mail: rector@rector.msu.su
Поступила в редакцию 25.10 .2006 и 23.07 .2007 